\title{
Database of Human Evaluations of Machine Translation Systems for Patent Translation
}

\author{
Isao Goto ${ }^{\dagger}$, Bin $\mathrm{Lu}^{\dagger \dagger, \dagger \dagger \dagger}$, Ka Po Chow ${ }^{\dagger \dagger}$, Eiichiro Sumita ${ }^{\dagger}$, \\ Benjamin K. Tsou ${ }^{\dagger \dagger} \dagger^{\dagger \dagger}$, Masao Utiyama ${ }^{\dagger}$ and Keiji Yasuda ${ }^{\dagger}$
}

This paper discusses a database of human evaluations of patent machine translation, from Chinese to English, Japanese to English, and English to Japanese. The evaluations were conducted for the NTCIR-9 Patent Machine Translation Task (PatentMT). Different types of systems, such as research systems and commercial systems, and rule-based systems and statistical machine translation systems were evaluated. Since human evaluation results are important when investigating automatic evaluation of translation quality, the database of the evaluation results is valuable. From the NTCIR project, resources including the human evaluation database, translation results, and test/reference data are available for research purposes.

Key Words: Human evaluation, Patent translation, Machine translation

\section{Introduction}

Automatic evaluation of translation quality is important for development of machine translation systems; thus, it is an active area of research (Papineni, Roukos, Ward, and Zhu 2002; Lin and Hovy 2003; Isozaki, Hirao, Duh, Sudoh, and Tsukada 2010a). Human evaluation resources, which can be used as fundamental data for the research, have been published. NIST Metrics for Machine Translation (MetricsMATR) ${ }^{1}$ published human evaluation resources for the news domain and for Arabic/Chinese-to-English translations. The Workshop on Statistical Machine Translation (WMT) ${ }^{2}$ (Callison-Burch, Koehn, Monz, Post, Soricut, and Specia 2012) published human evaluation resources for news and European Parliament Proceedings and translations between European languages.

The database of human evaluations that we introduce in this paper is different from the above-mentioned human evaluation resources in that it targets the patent domain and focuses on translations that include the Asian languages of Japanese and Chinese. This human evaluation

\footnotetext{
$\dagger$ National Institute of Information and Communications Technology

†† Hong Kong Institute of Education

†† City University of Hong Kong

${ }^{1}$ LDC Catalog No.: LDC2009T05 and LDC2011T05

2 http://www.statmt.org/wmt12/results.html
} 
Table 1 Comparison of NTCIR-7, 8, and 9.

\begin{tabular}{l|l|l|l}
\hline & \multicolumn{1}{|c|}{ NTCIR-7 } & \multicolumn{1}{c}{ NTCIR-8 } & \multicolumn{1}{c}{ NTCIR-9 } \\
\hline Language & $\begin{array}{l}\text { Japanese to English } \\
\text { English to Japanese }\end{array}$ & $\begin{array}{l}\text { Japanese to English } \\
\text { English to Japanese }\end{array}$ & $\begin{array}{l}\text { Chinese to English } \\
\text { Japanese to English } \\
\text { English to Japanese }\end{array}$ \\
\hline Human evaluation & Adequacy Fluency & No human evaluation & Adequacy Acceptability \\
\hline Extrinsic evaluation & Cross-lingual patent retrieval & Cross-lingual patent retrieval & No extrinsic evaluation \\
\hline Period & $7 / 2007$ to $12 / 2008$ & $1 / 2009$ to $6 / 2010$ & $7 / 2010$ to $12 / 2011$ \\
\hline
\end{tabular}

database was produced through the Patent Machine Translation Task (PatentMT) at NTCIR-9 ${ }^{3}$.

PatentMT at NTCIR-9 builds on the previous patent translation tasks performed at NTCIR-7 (Fujii, Utiyama, Yamamoto, and Utsuro 2008) and NTCIR-8 (Fujii, Utiyama, Yamamoto, Utsuro, Ehara, Echizen-ya, and Shimohata 2010) and contains two additions that were not present in the previous two tasks: Chinese-to-English translation and acceptability evaluations. The need to translate patent information from the Chinese language has increased, and therefore, a Chineseto-English subtask was added ${ }^{4}$. A comparison of NTCIR-7, 8, and 9 is summarized in Table 1. A human evaluation for acceptability was conducted, aiming to realize a more practical evaluation than is afforded by adequacy. The human evaluation reveals the number of test sentences that can be understood from the translations.

This paper ${ }^{5}$ gives a brief overview of NTCIR-9 PatentMT and describes the human evaluation results obtained at NTCIR-9 PatentMT. The effectiveness of the different state-of-the-art machine translation systems can be evaluated by comparing the human evaluation results of translations done by the different systems using the same test data. The database of the human evaluations is useful for research in the automatic evaluation of translation quality.

This paper is organized as follows: Section 2 explains the task design, Section 3 lists the participants and submissions, Section 4 describes the human evaluation results, Section 5 shows the validation of human evaluations, Section 6 gives a meta-evaluation of the automatic evaluation measure of BLEU, Section 7 shows the method for obtaining the database, and Section 8 concludes the paper.

\footnotetext{
3 The NTCIR-9 workshop was held December 6-9, 2011 in Tokyo. http://research.nii.ac.jp/ntcir/ntcir-9/

${ }^{4}$ We did not add the English-to-Chinese subtask because of three reasons: 1) Since human evaluation incurs significant costs, such as expense and labor, conducting human evaluations for four subtasks was difficult at that time. 2) We thought that the Chinese-to-English translation was more popular than the English-toChinese translation. 3) We could not provide a large-scale monolingual patent corpus in Chinese.

5 This paper is based on a presentation given at the NTCIR-9 workshop (Goto, Lu, Chow, Sumita, and Tsou 2011).
} 


\section{Task design}

PatentMT had three patent machine translation subtasks: Chinese to English (CE), Japanese to English (JE), and English to Japanese (EJ). Participants chose the subtasks in which they wished to participate and were provided with training data, development data, and test data. Participants translated the test data using their machine translation systems and submitted the translations to the PatentMT organizers. The PatentMT organizers evaluated the submitted translations and returned the evaluation results to the participants. Finally, the participants presented their research results at the NTCIR-9 workshop.

\subsection{Data provided to the participants}

The provided data consisted of training data, development data, test data, context documents, and reference data. The reference data was provided after the submission of translation results. The training data consisted of a parallel corpus and a monolingual corpus. The parallel sentence pairs for the training, development, and test/reference data were drawn from patent description sentences (patent documents consist of a title, abstract, claim, and description). The parallel sentence pairs for the training data were automatically extracted from patent documents using bilingual dictionaries. The Chinese-English parallel sentence pairs were extracted from Patent Cooperation Treaty (PCT) patents in Chinese and English (Lu, Tsou, Jiang, Kwong, and Zhu 2010). The Japanese-English parallel sentence pairs were extracted from the patent family in Japanese and English (Utiyama and Isahara 2007). The training data was built from patent documents published between 1993 and 2005. The number of patent parallel sentence pairs for the training data was: 1 million for Chinese-English and approximately 3.2 million for JapaneseEnglish. The training data of the monolingual corpus was a monolingual patent corpus in the target language spanning 13 years (1993-2005). The test data was built by randomly selecting parallel sentences from a portion of the automatically built patent parallel sentence pairs published in 2006 and 2007, manually judging whether the sentence pairs were correct translations, then selecting 2,000 correct sentence pairs as the test data and their reference data. The patent documents from which the test sentences were extracted were provided as context documents for the test data.

\subsection{Evaluation methodology}

We conducted human evaluations and regarded these as the primary evaluation. 
Human evaluations were carried out by paid evaluation experts ${ }^{6}$ and employed the criteria of adequacy and acceptability, which will be explained later. For each criterion, three evaluators evaluated 100 sentences per system. The three evaluators evaluated different sentences. Thus, 300 sentences were evaluated per system. The 300 sentences were randomly selected from the test sentences. In this evaluation, the evaluators looked at a source sentence and its translation results to be evaluated.

\subsubsection{Adequacy}

We conducted a 5 -scale ( 1 to 5 ) adequacy evaluation. The main purpose of the adequacy evaluation was to compare the systems.

Adequacy can be defined in multiple ways. White (White, O'Connell, and O'Mara 1994) defined it as how much of the information from a fragment of a reference sentence is contained in the translation results. They insisted that fragmentation is intended to avoid biasing the results in favor of linguistic compositional approaches (which may do relatively better on longer, clauselevel strings) or statistical approaches (which may do better on shorter strings not associated with syntactic constituency). However, this evaluation cannot evaluate whether the sentence meaning is correct or not because simply containing all of the fragments of the reference information does not guarantee a correct sentence meaning. The NTCIR-7 Patent Translation Task (Fujii et al. 2008) conducted adequacy evaluations using a criterion based on the degree of preservation of sentence-level meaning instead of the degree of fragments of the reference information contained.

We believed that the degree of sentence-level meaning preservation was better than that of fragments of reference information contained for the evaluation of translation quality. However, since the cost of checking sentence meanings is high, we evaluated quality considering the clauselevel meanings for adequacy.

The instructions for the adequacy criterion are given in Appendix A.1. Examples of adequacy values and translations are shown in Appendix A.2.

The systems were ranked based on adequacy using the average system scores.

\footnotetext{
${ }^{6}$ Because we evaluated machine translations and not human translations, we did not perform evaluations in a similar manner as human translations. The evaluators were not patent experts for the domains. This evaluation did not check whether the translations of technical terms were perfect. The writing style for the test data is a general style because all of the test sentences were from description sections of patents and were not from claim sections. Therefore, the evaluators, who are not patent experts, can distinguish whether a translated sentence represents the source sentence meaning. The evaluator profiles were as follows: For CE, adequacy: Chinese native speakers who can understand English; acceptability: Chinese native speakers whose English abilities are very high. For JE, adequacy and acceptability: English native speakers who can understand Japanese. For EJ, adequacy and acceptability: Japanese native speakers who can understand English.
} 


\subsubsection{Acceptability}

We conducted a 5-scale acceptability evaluation as shown in Fig. 1. The main purpose of an acceptability evaluation is to clarify the percentage of translated sentences for which the source sentence meanings can be understood from randomly selected test sentences. ${ }^{7}$ Acceptability is an evaluation of sentence-level meaning. The acceptability criterion used in this evaluation is aimed more at practical evaluation as opposed to adequacy. For example, if the requirement of a translation system is that the source sentence meaning can be understood, translations of $\mathrm{C}$ or higher are useful; however, if the requirement is that the source sentence meaning can be understood and the sentence is grammatically correct, then only translations of A or higher are useful. We can then know the number of sentences from a system would be useful for each requirement. An adequacy criterion cannot answer these requirements.

Acceptability also contains an evaluation of fluency that measures fluency in the target language, since it also affects the differences in grading from $\mathrm{C}$ to AA. If the adequacy of a translation is very low, then the translation is not correct even if the fluency is high. If the integrated evaluation score is calculated by averaging the adequacy and fluency scores, then those translations

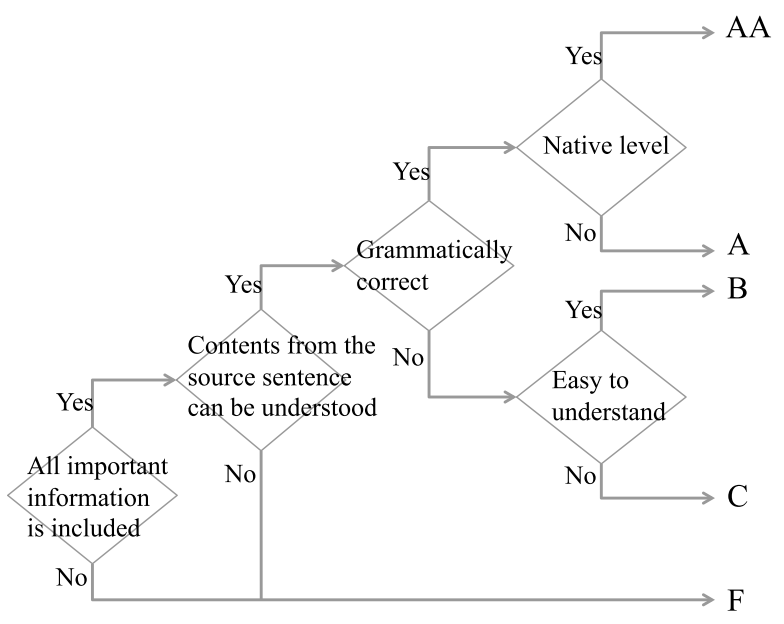

Fig. 1 Acceptability

\footnotetext{
7 Informative, shown in the ALPAC report (Pierce, Carroll, Hamp, Hays, Hockett, Oettinger, and Perlis 1966) p.70, is also an evaluation criterion of translation quality. Informative is a measure of how informative the original version is perceived to be after the translation has been seen using a scale from 0 to 9 . The upper grades are decided on the basis of whether a translation includes word-level or sentence structure-level errors. Because there are cases where the source sentence meaning can be understood as well as cases where it cannot be understood when a translation includes sentence structure-level errors, informative cannot clarify the difference in understandability. In contrast, acceptability can judge whether the source sentence meaning can be understood independent of the existence of word-level or sentence-structure-level errors.
} 
could be overvalued. Acceptability avoids this problem, allowing us to consider fluency.

The instructions for the acceptability criterion are shown in Appendix B.1. Examples of acceptability values and translations are shown in Appendix B.2.

We ranked the systems based on acceptability using a pairwise comparison, which will now be explained. The pairwise score for a system A reflects how frequently it was judged to be better than or equal to other systems. Suppose there are five systems to be compared. For each input sentence, system A is included in four pairwise comparisons (against the other systems). System A is rewarded as 1.0 for each of the comparisons in which system A is ranked the highest of the two, and 0.5 for each of the comparisons in which system A is in a tie. System A's score is the total rewarded score in the pairwise comparisons divided by the total number of pairwise comparisons involving system A.

Note that the average score of acceptability was not used for system ranking. The reason is as follows. Here we assume that the differences between the grades are measured by general usability. It is important to be able to understand the contents from the source sentence. There is a large difference in usability between $\mathrm{F}$ and $\mathrm{C}$. However, at the A-level, while the translations are at a non-native level, the contents from the source sentences can be understood and they are grammatically correct; thus, they have the potential to be useful in many cases. Thus, it is believed that the difference in usability between $\mathrm{A}$ and $\mathrm{AA}$ is smaller than that between $\mathrm{F}$ and C. In addition, we think that useful grades depend on specific usage. Therefore, it is difficult to give an appropriate score for each grade, and we avoided the conversion of grades to scores and calculation of averages.

\subsubsection{Human evaluation procedure}

We conducted human evaluation training before the main evaluation to normalize the evaluators' criteria. In the training, all evaluators evaluated 100 translations, and a meeting was held to determine common results for each subtask. The main evaluation was then performed. The common results produced at the training were used as the reference results for the main evaluation.

The instructions for the human evaluation procedure are shown in Appendix C.

\subsection{Schedule}

Translations were done over a two-week period in May 2011. 


\section{Participants and submissions}

We received submissions from 21 groups. The number of groups for each subtask was: 18 for CE, 12 for JE, and 9 for EJ. Table 2 shows the Group IDs, the participant organizations, system description papers, and the subtasks in which they participated. The types of translation systems are statistical machine translation (SMT), rule-based machine translation (RBMT), examplebased machine translation (EBMT), or hybrids of two or more types (HYBRID).

In addition to the submissions from the participants, the organizers submitted results for

Table 2 Participants and subtasks participated in.

\begin{tabular}{|c|c|c|c|c|}
\hline \multirow{2}{*}{ Group ID } & \multirow{2}{*}{ Participant } & \multicolumn{3}{|c|}{ Subtasks } \\
\hline & & $\mathrm{CE}$ & JE & EJ \\
\hline EIWA & Yamanashi Eiwa College (Ehara 2011) & $\checkmark$ & $\checkmark$ & \\
\hline KLE & $\begin{array}{l}\text { Pohang University of Science and Technology (POSTECH) (Na } \\
\text { et al. 2011) }\end{array}$ & $\checkmark$ & $\checkmark$ & $\checkmark$ \\
\hline TORI & Tottori University (Murakami and Tokuhisa 2011) & & $\checkmark$ & $\checkmark$ \\
\hline RWTH & RWTH Aachen University (Feng et al. 2011) & $\checkmark$ & $\checkmark$ & \\
\hline FRDC & Fujitsu R\&D Center CO., LTD (Zheng et al. 2011) & $\checkmark$ & $\checkmark$ & $\checkmark$ \\
\hline $\mathrm{NEU}$ & Northeastern University (Xiao et al. 2011) & $\checkmark$ & $\checkmark$ & \\
\hline BUAA & $\begin{array}{l}\text { Institute of Intelligent Information Processing, Beihang University } \\
\text { (Chao and Li 2011) }\end{array}$ & $\checkmark$ & & \\
\hline UOTTS & The University of Tokyo (Wu et al. 2011) & $\checkmark$ & $\checkmark$ & $\checkmark$ \\
\hline NCW & $\begin{array}{l}\text { NTNU, NCCU, and WebGenie Information Ltd. (Tseng et al. } \\
\text { 2011) }\end{array}$ & $\checkmark$ & & \\
\hline ICT & $\begin{array}{l}\text { Institute of Computing Technology, Chinese Academy of Sciences } \\
\text { (Xiong et al. 2011) }\end{array}$ & $\checkmark$ & $\checkmark$ & $\checkmark$ \\
\hline BJTUX & Beijing Jiaotong University (Jiang et al. 2011) & $\checkmark$ & & $\checkmark$ \\
\hline $\mathrm{BBN}$ & Raytheon BBN Technologies (Ma and Matsoukas 2011) & $\checkmark$ & & \\
\hline NAIST & Nara Institute of Science and Technology (Kondo et al. 2011) & & $\checkmark$ & \\
\hline IBM & IBM Research (Lee et al. 2011) & $\checkmark$ & & \\
\hline KECIR & Shenyang Aerospace University & $\checkmark$ & & \\
\hline JAPIO & Japan Patent Information Organization (Ohio et al. 2011) & & $\checkmark$ & $\checkmark$ \\
\hline KYOTO & Kyoto University (Nakazawa and Kurohashi 2011) & $\checkmark$ & $\checkmark$ & $\checkmark$ \\
\hline NTHU (IDEAS) & $\begin{array}{l}\text { Institute for Information Industry, Chaoyang University of Tech- } \\
\text { nology and National Tsing Hua University (Chang et al. 2011) }\end{array}$ & $\checkmark$ & & \\
\hline NTT-UT & $\begin{array}{l}\text { NTT Communication Science Labs and the University of Tokyo } \\
\text { (Sudoh et al. 2011) }\end{array}$ & $\checkmark$ & $\checkmark$ & $\checkmark$ \\
\hline ISTIC & $\begin{array}{l}\text { Institute of Scientific and Technical Information of China (He et al. } \\
\text { 2011) }\end{array}$ & $\checkmark$ & & \\
\hline LIUM & University of Le Mans (Schwenk and Abdul-Rauf 2011) & $\checkmark$ & & \\
\hline
\end{tabular}


baseline systems that consisted of 2 SMT systems, 5 commercial RBMT systems, and 1 online SMT system. The baseline systems are shown in Table 3. The SMT baseline systems consisted of publicly available software, and the procedures for building the systems and translating using the systems were published on the PatentMT web page ${ }^{8}$ so that those with the training data can build the SMT baseline systems and compare their results. The commercial RBMT systems and the Google online translation system ${ }^{9}$ were operated by the organizers. The translation results from the Google translation system were created by translating the test data via their web interface. We note that these RBMT companies and Google did not submit themselves. Since our objective does not include comparing the commercial RBMT systems of companies who did not themselves participate, the System IDs of the commercial RBMT systems are kept anonymous in this paper.

Each participant is allowed to submit as many translated results ("runs") as desired, but the submitted runs should be prioritized by the group. In this paper, we distinguish their runs using a Run ID expressed by Group ID (or System ID for the baseline systems) and a priority number connected by "_". The resource information used by each run is indicated by

- Resource B: The system used the bilingual training data provided by the organizers.

- Resource $M$ : The system used the monolingual training data provided by the organizers.

- Resource E: The system used external knowledge other than data provided by the organizers or the system uses a rule-based system.

Table 3 Baseline systems.

\begin{tabular}{l|l|l|l|l|l}
\hline \multicolumn{1}{c|}{ System ID } & \multicolumn{1}{|c|}{ System } & Type & CE & JE & EJ \\
\hline BASELINE1 & Moses' hierarchical phrase-based SMT system (Hoang et al. & SMT & $\checkmark$ & $\checkmark$ & $\checkmark$ \\
& 2009) & & & & \\
BASELINE2 & Moses' phrase-based SMT system (Koehn et al. 2007) & SMT & $\checkmark$ & $\checkmark$ & $\checkmark$ \\
RBMTx & SYSTRAN 7 Premium Translator (Commercial RBMT) & RBMT & $\checkmark$ & & \\
RBMTx & Huajian Multilingual EasyTrans version 3.0 (Commercial & RBMT & $\checkmark$ & & \\
& RBMT) & & & & \\
RBMTx & The Honyaku 2009 premium patent edition (Commercial & RBMT & & $\checkmark$ & $\checkmark$ \\
& RBMT) & & & & \\
RBMTx & ATLAS V14 (Commercial RBMT) & & $\checkmark$ & $\checkmark$ \\
RBMTx & PAT-Transer 2009 (Commercial RBMT) & & \\
ONLINE1 1 & Google online translation system (June, 2011) & & $\checkmark$ & $\checkmark$ \\
\hline
\end{tabular}

\footnotetext{
8 http://ntcir.nii.ac.jp/PatentMT/

9 http://www.google.com
} 


\section{Human evaluation results}

We evaluated the adequacy for at least all of the first priority submissions. However, because of budget limitations, acceptability was evaluated for only selected systems.

\subsection{Chinese to English}

\subsubsection{Adequacy evaluation}

Table 4 shows the results of the adequacy evaluation. Table 5 shows the results of the statistical significance test of the adequacy evaluation using a sign test. In the tables showing the results of a statistical significance test, the marks (">”, ">”, "“") indicate whether the Run ID to the left of a mark is significantly better than that above the mark.

From these results, we can observe the following:

- All of the top systems are SMT systems. The top system, BBN-1, shows a significantly higher adequacy than the other systems.

Table 4 Results of CE adequacy.

\begin{tabular}{|c|c|c|c|c|c|c|c|c|c|c|}
\hline \multirow{2}{*}{ Run ID } & \multirow{2}{*}{ Type } & \multicolumn{3}{|c|}{ Resource } & \multirow{2}{*}{$\begin{array}{c}\text { Average } \\
\text { score }\end{array}$} & \multicolumn{5}{|c|}{ Rate } \\
\hline & & B & $\mathrm{M}$ & $\mathrm{E}$ & & 5 & 4 or higher & 3 or higher & 2 or higher & 1 or higher \\
\hline BBN-1 & SMT & $\checkmark$ & $\checkmark$ & & 4.033 & 0.397 & 0.700 & 0.940 & 0.997 & 1.000 \\
\hline NEU-1 & SMT & $\checkmark$ & $\checkmark$ & & 3.510 & 0.237 & 0.460 & 0.827 & 0.987 & 1.000 \\
\hline RWTH-1 & SMT & $\checkmark$ & $\checkmark$ & & 3.420 & 0.210 & 0.417 & 0.797 & 0.997 & 1.000 \\
\hline LIUM-1 & SMT & $\checkmark$ & $\checkmark$ & & 3.403 & 0.193 & 0.420 & 0.800 & 0.990 & 1.000 \\
\hline IBM-1 & SMT & $\checkmark$ & $\checkmark$ & $\checkmark$ & 3.390 & 0.170 & 0.447 & 0.793 & 0.980 & 1.000 \\
\hline FRDC-1 & SMT & $\checkmark$ & $\checkmark$ & $\checkmark$ & 3.340 & 0.177 & 0.380 & 0.790 & 0.993 & 1.000 \\
\hline KLE-1 & SMT & $\checkmark$ & & & 3.340 & 0.177 & 0.377 & 0.807 & 0.980 & 1.000 \\
\hline ICT-1 & $\mathrm{SMT}$ & $\checkmark$ & $\checkmark$ & & 3.300 & 0.150 & 0.370 & 0.787 & 0.993 & 1.000 \\
\hline BUAA-1 & HYBRID & $\checkmark$ & $\checkmark$ & & 3.297 & 0.113 & 0.367 & 0.833 & 0.983 & 1.000 \\
\hline UOTTS-1 & $\mathrm{SMT}$ & $\checkmark$ & & & 3.293 & 0.123 & 0.363 & 0.817 & 0.990 & 1.000 \\
\hline BASELINE1-1 & SMT & $\checkmark$ & & & 3.290 & 0.160 & 0.373 & 0.780 & 0.977 & 1.000 \\
\hline NTT-UT-1 & SMT & $\checkmark$ & & & 3.230 & 0.103 & 0.343 & 0.797 & 0.987 & 1.000 \\
\hline ISTIC-1 & HYBRID & $\checkmark$ & $\checkmark$ & & 3.187 & 0.107 & 0.333 & 0.767 & 0.980 & 1.000 \\
\hline NTHU-1 & $\mathrm{SMT}$ & $\checkmark$ & $?$ & $\checkmark$ & 3.127 & 0.123 & 0.320 & 0.710 & 0.973 & 1.000 \\
\hline BJTUX-1 & SMT & $\checkmark$ & & & 3.113 & 0.103 & 0.310 & 0.723 & 0.977 & 1.000 \\
\hline EIWA-1 & HYBRID & $\checkmark$ & & $\checkmark$ & 3.047 & 0.087 & 0.267 & 0.710 & 0.983 & 1.000 \\
\hline KECIR-1 & SMT & $\checkmark$ & $?$ & $\checkmark$ & 3.037 & 0.093 & 0.263 & 0.707 & 0.973 & 1.000 \\
\hline ONLINE1-1 & SMT & & & $\checkmark$ & 2.967 & 0.070 & 0.273 & 0.670 & 0.953 & 1.000 \\
\hline BASELINE2-1 & SMT & $\checkmark$ & & & 2.893 & 0.133 & 0.240 & 0.550 & 0.970 & 1.000 \\
\hline NCW-1 & SMT & $\checkmark$ & & & 2.853 & 0.077 & 0.200 & 0.600 & 0.977 & 1.000 \\
\hline RBMT2-1 & RBMT & & & $\checkmark$ & 2.663 & 0.033 & 0.170 & 0.523 & 0.937 & 1.000 \\
\hline KYOTO-1 & EBMT & $\checkmark$ & & & 2.410 & 0.037 & 0.130 & 0.407 & 0.837 & 1.000 \\
\hline RBMT1-1 & RBMT & & & $\checkmark$ & 2.277 & 0.043 & 0.097 & 0.353 & 0.783 & 1.000 \\
\hline
\end{tabular}


Table 5 Sign test of CE adequacy. "》": significantly different at $\alpha=0.01$, , $>$ ": significantly different at $\alpha=0.05$, "-": not significantly different at $\alpha=0.05$.

\begin{tabular}{|c|c|c|c|c|c|c|c|c|c|c|c|c|c|c|c|c|c|c|c|c|c|c|c|}
\hline & 空 & 要 & 每 & & $\sum_{\theta}^{I}$ & 岳 & 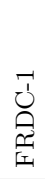 & 易 & 安 & 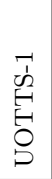 & $\begin{array}{l}\frac{1}{1} \\
\text { 至 } \\
\text { 至 } \\
\text { 昂 }\end{array}$ & 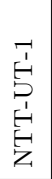 & 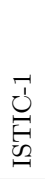 & $\begin{array}{l}\overrightarrow{3} \\
\text { 䃾 }\end{array}$ & 离 & 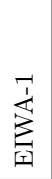 & 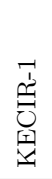 & $\begin{array}{l}\frac{7}{1} \\
\text { 空 } \\
\text { 号 } \\
0\end{array}$ & 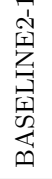 & $\begin{array}{l}\overline{1} \\
0 \\
z \\
z\end{array}$ & 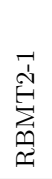 & \begin{tabular}{l}
$\overrightarrow{1}$ \\
0 \\
0 \\
0 \\
\multirow{1}{*}{}
\end{tabular} & $\stackrel{\vec{E}}{\vec{E}}$ \\
\hline $\mathrm{BBN}-1$ & $\gg$ & $\gg$ & $\geq$ & & $\gg$ & $\gg$ & $\gg$ & $\gg$ & $\gg$ & $\gg$ & $\gg$ & $\gg$ & $\gg$ & $\gg$ & $\gg$ & $\gg$ & $\gg$ & $\gg$ & $\gg$ & $\gg$ & $\gg$ & $\gg$ & $\gg$ \\
\hline NEU-1 & & - & > & & - & $>$ & $\gg$ & $\gg$ & $\gg$ & $\gg$ & $\gg$ & $\gg$ & $\gg$ & $\gg$ & $\gg$ & $\gg$ & $\gg$ & $\gg$ & $\gg$ & $\gg$ & $\gg$ & $\gg$ & $\gg$ \\
\hline RWTH-1 & & & - & & - & - & - & - & - & $>$ & - & $\gg$ & $\gg$ & $\gg$ & $\gg$ & $\gg$ & $\gg$ & $\gg$ & $\gg$ & $\gg$ & $\gg$ & $\gg$ & $\gg$ \\
\hline LIUM-1 & & & & & - & - & - & - & - & - & - & $\gg$ & $\gg$ & $\gg$ & $\gg$ & $\gg$ & $\gg$ & $\gg$ & $\gg$ & $\gg$ & $\gg$ & $\gg$ & $\gg$ \\
\hline IBM-1 & & & & & & - & - & - & - & - & - & $>$ & $\gg$ & $\gg$ & $\gg$ & $\gg$ & $\gg$ & $\gg$ & $\gg$ & $\gg$ & $\gg$ & $\gg$ & $\gg$ \\
\hline KLE-1 & & & & & & & - & - & - & - & - & - & $\gg$ & $\gg$ & $\gg$ & $\gg$ & $\gg$ & $\gg$ & $\gg$ & $\gg$ & $\gg$ & $\gg$ & $\gg$ \\
\hline FRDC-1 & & & & & & & & - & - & - & - & - & $\gg$ & $\gg$ & $\gg$ & $\gg$ & $\gg$ & $\gg$ & $\gg$ & $\gg$ & $\gg$ & $\gg$ & $\gg$ \\
\hline ICT-1 & & & & & & & & & - & - & - & - & $>$ & $\gg$ & $\gg$ & $\gg$ & $\gg$ & $\gg$ & $\gg$ & $\gg$ & $\gg$ & $\gg$ & $\gg$ \\
\hline BUAA-1 & & & & & & & & & & - & - & - & - & $\gg$ & $>$ & $\gg$ & $\gg$ & $\gg$ & $\gg$ & $\gg$ & $\gg$ & $\gg$ & $\gg$ \\
\hline UOTTS-1 & & & & & & & & & & & - & - & $\gg$ & $\gg$ & $>$ & $\gg$ & $\gg$ & $\gg$ & $\gg$ & $\gg$ & $\gg$ & $\gg$ & $\gg$ \\
\hline BASELINE1-1 & & & & & & & & & & & & - & - & $>$ & $>$ & $\gg$ & $\gg$ & $\gg$ & $\gg$ & $\gg$ & $\gg$ & $\gg$ & $\gg$ \\
\hline NTT-UT-1 & & & & & & & & & & & & & - & - & - & $\gg$ & $\gg$ & $\gg$ & $\gg$ & $\gg$ & $\gg$ & $\gg$ & \\
\hline ISTIC-1 & & & & & & & & & & & & & & - & - & $>$ & $\gg$ & $\gg$ & $\gg$ & $\gg$ & $\gg$ & $\gg$ & \\
\hline NTHU-1 & & & & & & & & & & & & & & & - & - & - & $\gg$ & $\gg$ & $\gg$ & $\gg$ & $\gg$ & \\
\hline BJTUX-1 & & & & & & & & & & & & & & & & - & - & $\gg$ & $\gg$ & $\gg$ & $\gg$ & $\gg$ & \\
\hline EIWA-1 & & & & & & & & & & & & & & & & & - & - & $\gg$ & $\gg$ & $\gg$ & $\gg$ & \\
\hline KECIR-1 & & & & & & & & & & & & & & & & & & - & $\gg$ & $\gg$ & $\gg$ & $\gg$ & \\
\hline ONLINE1-1 & & & & & & & & & & & & & & & & & & & - & $>$ & $\gg$ & $\gg$ & \\
\hline BASELINE2-1 & & & & & & & & & & & & & & & & & & & & - & $>$ & $\gg$ & $\gg$ \\
\hline NCW-1 & & & & & & & & & & & & & & & & & & & & & $\gg$ & $\gg$ & $\gg$ \\
\hline RBMT2-1 & & & & & & & & & & & & & & & & & & & & & & $\gg$ & $\gg$ \\
\hline KYOTO-1 & & & & & & & & & & & & & & & & & & & & & & & - \\
\hline
\end{tabular}

- The adequacy score for Moses' hierarchical phrase-based SMT system (BASELINE1-1) is higher than that for Moses' phrase-based SMT system (BASELINE2-1).

- The adequacy scores for Moses' hierarchical phrase-based SMT system (BASELINE1-1) and Moses' phrase-based SMT system (BASELINE2-1) are higher than those for the two RBMT baseline systems (RBMT2-1 and RBMT1-1).

To improve translation quality, the top BBN-1 system (Ma and Matsoukas 2011) used the following techniques: generalization of infrequent numerical expressions, optimization of Chinese word segmentation, adaptation of language models, addition of features, and utilization of English dependency structures. Effectiveness of the system using these techniques was shown.

\subsubsection{Acceptability evaluation}

Table 6 shows the results of the acceptability evaluation. Table 7 shows the results of the statistical significance test of the acceptability evaluation using a sign test. 
Table 6 Results of CE acceptability.

\begin{tabular}{|c|c|c|c|c|c|c|c|c|c|c|}
\hline \multirow{2}{*}{ Run ID } & \multirow{2}{*}{ Type } & \multicolumn{3}{|c|}{ Resource } & \multirow{2}{*}{$\begin{array}{c}\text { Pairwise } \\
\text { score }\end{array}$} & \multicolumn{5}{|c|}{ Rate } \\
\hline & & $\mathrm{B}$ & $\mathrm{M}$ & $\mathrm{E}$ & & $\mathrm{AA}$ & A or higher & $\mathrm{B}$ or higher & $\mathrm{C}$ or higher & F or higher \\
\hline BBN-1 & SMT & $\checkmark$ & $\checkmark$ & & 0.744 & 0.143 & 0.403 & 0.627 & 0.797 & 1.000 \\
\hline RWTH-1 & SMT & $\checkmark$ & $\checkmark$ & & 0.546 & 0.087 & 0.217 & 0.357 & 0.540 & 1.000 \\
\hline NEU-1 & SMT & $\checkmark$ & $\checkmark$ & & 0.544 & 0.083 & 0.193 & 0.407 & 0.557 & 1.000 \\
\hline IBM-1 & SMT & $\checkmark$ & $\checkmark$ & $\checkmark$ & 0.513 & 0.083 & 0.187 & 0.343 & 0.507 & 1.000 \\
\hline LIUM-1 & SMT & $\checkmark$ & $\checkmark$ & & 0.508 & 0.063 & 0.193 & 0.333 & 0.510 & 1.000 \\
\hline FRDC-1 & SMT & $\checkmark$ & $\checkmark$ & $\checkmark$ & 0.495 & 0.050 & 0.153 & 0.343 & 0.500 & 1.000 \\
\hline KLE-1 & $\mathrm{SMT}$ & $\checkmark$ & & & 0.491 & 0.073 & 0.160 & 0.317 & 0.470 & 1.000 \\
\hline BUAA-1 & HYBRID & $\checkmark$ & $\checkmark$ & & 0.486 & 0.030 & 0.107 & 0.317 & 0.517 & 1.000 \\
\hline BASELINE1-1 & $\mathrm{SMT}$ & $\checkmark$ & & & 0.476 & 0.047 & 0.143 & 0.293 & 0.473 & 1.000 \\
\hline ICT-1 & $\mathrm{SMT}$ & $\checkmark$ & $\checkmark$ & & 0.468 & 0.050 & 0.143 & 0.290 & 0.447 & 1.000 \\
\hline UOTTS-1 & SMT & $\checkmark$ & & & 0.441 & 0.027 & 0.113 & 0.287 & 0.427 & 1.000 \\
\hline ONLINE1-1 & SMT & & & $\checkmark$ & 0.422 & 0.040 & 0.083 & 0.210 & 0.420 & 1.000 \\
\hline RBMT2-1 & RBMT & & & $\checkmark$ & 0.365 & 0.003 & 0.033 & 0.160 & 0.340 & 1.000 \\
\hline
\end{tabular}

Table 7 Sign test of CE acceptability. "\$": significantly different at $\alpha=0.01$, ">": significantly different at $\alpha=0.05$, "”": not significantly different at $\alpha=0.05$.

\begin{tabular}{|c|c|c|c|c|c|c|c|c|c|c|c|c|}
\hline & 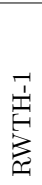 & 忿 & $\sum_{\ominus}^{+}$ & $\underset{⿱ 乛}{\stackrel{3}{S}}$ & 苞 & 岳 & 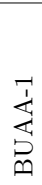 & 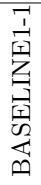 & $\underbrace{-1}$ & \begin{tabular}{l}
7 \\
\multicolumn{1}{c}{} \\
0 \\
0 \\
0 \\
0 \\
0
\end{tabular} & $\begin{array}{l}\frac{7}{1} \\
\text { 吾 } \\
\text { 点 } \\
\text { 点 }\end{array}$ & 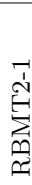 \\
\hline BBN-1 & $\gg$ & $\gg$ & $\gg$ & $\gg$ & $\gg$ & $\gg$ & $\gg$ & $\gg$ & $\gg$ & $\gg$ & $\gg$ & $\gg$ \\
\hline RWTH-1 & & - & - & $>$ & $\gg$ & $>$ & $\gg$ & $>$ & $\gg$ & $\gg$ & $\gg$ & $\gg$ \\
\hline NEU-1 & & & - & - & $>$ & $>$ & $>$ & $\gg$ & $\gg$ & $\gg$ & $\gg$ & $\gg$ \\
\hline IBM-1 & & & & - & - & - & - & - & $>$ & $\gg$ & $\gg$ & $\gg$ \\
\hline LIUM-1 & & & & & - & - & - & - & - & $\gg$ & $\gg$ & $\gg$ \\
\hline FRDC-1 & & & & & & - & - & - & - & $\gg$ & $\gg$ & $\gg$ \\
\hline KLE-1 & & & & & & & - & - & - & $\gg$ & $\gg$ & $\gg$ \\
\hline BUAA-1 & & & & & & & & - & - & - & $\gg$ & $\gg$ \\
\hline BASELINE1-1 & & & & & & & & & - & - & $>$ & $\gg$ \\
\hline ICT-1 & & & & & & & & & & $>$ & $>$ & $\gg$ \\
\hline UOTTS-1 & & & & & & & & & & & - & $\gg$ \\
\hline ONLINE1-1 & & & & & & & & & & & & $\gg$ \\
\hline
\end{tabular}

From the results, we can see that the meaning of the source language could be understood (C-rank and above) for $79.7 \%$ of the translated sentences in the best-ranked system (BBN-1). This result significantly surpasses the others. 


\subsection{Japanese to English}

\subsubsection{Adequacy evaluation}

Table 8 shows the results of the adequacy evaluation. Table 9 shows the results of the statistical significance test of the adequacy evaluation using a sign test.

The top five systems, JAPIO-1, RBMT1-1, EIWA-1, RBMT3-1, and RBMT2-1, are either commercial RBMT systems or systems that use commercial RBMT systems. From these results, the following are observed:

- The commercial RBMT systems had higher adequacies than the state-of-the-art SMT systems for patent machine translation from Japanese to English.

- The adequacy score for Moses' hierarchical phrase-based SMT (BASELINE1-1) is slightly higher than that for Moses' phrase-based SMT (BASELINE2-1).

The reason that the SMT systems could not achieve adequacy scores as high as those from the top RBMT systems is thought to be because of word ordering. Since the word order in Japanese and English is significantly different (Japanese is a Subject-Object-Verb (SOV) language and English is a Subject-Verb-Object (SVO) language), word ordering is difficult for Japanese-English translation. The current SMT performs well for word selection, but not for difficult word ordering

Table 8 Results of JE adequacy.

\begin{tabular}{|c|c|c|c|c|c|c|c|c|c|c|}
\hline \multirow{2}{*}{ Run ID } & \multirow{2}{*}{ Type } & \multicolumn{3}{|c|}{ Resource } & \multirow{2}{*}{$\begin{array}{c}\text { Average } \\
\text { score }\end{array}$} & \multicolumn{5}{|c|}{ Rate } \\
\hline & & B & M & $\mathrm{E}$ & & 5 & 4 or higher & 3 or higher & 2 or higher & 1 or higher \\
\hline JAPIO-1 & RBMT & & & $\checkmark$ & 3.667 & 0.297 & 0.590 & 0.807 & 0.973 & 1.000 \\
\hline RBMT1-1 & RBMT & & & $\checkmark$ & 3.530 & 0.273 & 0.530 & 0.763 & 0.963 & 1.000 \\
\hline EIWA-1 & HYBRID & $\checkmark$ & & $\checkmark$ & 3.430 & 0.260 & 0.473 & 0.727 & 0.970 & 1.000 \\
\hline RBMT3-1 & RBMT & & & $\checkmark$ & 3.137 & 0.183 & 0.393 & 0.623 & 0.937 & 1.000 \\
\hline RBMT2-1 & RBMT & & & $\checkmark$ & 3.073 & 0.190 & 0.377 & 0.597 & 0.910 & 1.000 \\
\hline NTT-UT-1 & SMT & $\checkmark$ & & & 2.747 & 0.117 & 0.260 & 0.457 & 0.913 & 1.000 \\
\hline TORI-1 & HYBRID & $\checkmark$ & $\checkmark$ & $\checkmark$ & 2.730 & 0.100 & 0.217 & 0.490 & 0.923 & 1.000 \\
\hline RWTH-1 & $\mathrm{SMT}$ & $\checkmark$ & & & 2.663 & 0.107 & 0.237 & 0.407 & 0.913 & 1.000 \\
\hline BASELINE1-1 & $\mathrm{SMT}$ & $\checkmark$ & & & 2.617 & 0.083 & 0.200 & 0.403 & 0.930 & 1.000 \\
\hline NAIST-1 & SMT & $\checkmark$ & & & 2.610 & 0.097 & 0.213 & 0.417 & 0.883 & 1.000 \\
\hline FRDC-1 & $\mathrm{SMT}$ & $\checkmark$ & $\checkmark$ & & 2.517 & 0.077 & 0.170 & 0.360 & 0.910 & 1.000 \\
\hline BASELINE2-1 & SMT & $\checkmark$ & & & 2.427 & 0.083 & 0.160 & 0.333 & 0.850 & 1.000 \\
\hline KYOTO-2 & SMT & $\checkmark$ & & & 2.413 & 0.057 & 0.117 & 0.350 & 0.890 & 1.000 \\
\hline KYOTO-1 & EBMT & $\checkmark$ & & & 2.380 & 0.060 & 0.160 & 0.307 & 0.853 & 1.000 \\
\hline UOTTS-1 & SMT & $\checkmark$ & & & 2.377 & 0.067 & 0.130 & 0.307 & 0.873 & 1.000 \\
\hline NEU-1 & SMT & $\checkmark$ & $\checkmark$ & & 2.373 & 0.033 & 0.127 & 0.317 & 0.897 & 1.000 \\
\hline ONLINE1-1 & $\mathrm{SMT}$ & & & $\checkmark$ & 2.273 & 0.050 & 0.103 & 0.250 & 0.870 & 1.000 \\
\hline ICT-1 & $\mathrm{SMT}$ & $\checkmark$ & $\checkmark$ & & 2.267 & 0.027 & 0.100 & 0.260 & 0.880 & 1.000 \\
\hline KLE-1 & SMT & $\checkmark$ & & & 2.040 & 0.037 & 0.117 & 0.193 & 0.693 & 1.000 \\
\hline
\end{tabular}


Table 9 Sign test of JE adequacy. "\$": significantly different at $\alpha=0.01$, , ": significantly different at $\alpha=0.05$, "-": not significantly different at $\alpha=0.05$.

\begin{tabular}{|c|c|c|c|c|c|c|c|c|c|c|c|c|c|c|c|c|c|c|}
\hline & 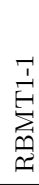 & 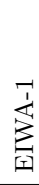 & 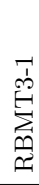 & 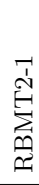 & 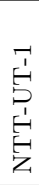 & 站 & 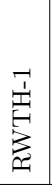 & 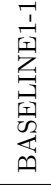 & $\begin{array}{l}\vec{H} \\
\text { 离 } \\
\text { 至 }\end{array}$ & $\begin{array}{l}\overrightarrow{0} \\
\overrightarrow{0} \\
\overrightarrow{\mid c}\end{array}$ & $\begin{array}{l}\text { 絰 } \\
\text { 空 } \\
\text { 命 }\end{array}$ & 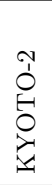 & $\begin{array}{l}0 \\
0 \\
0 \\
0 \\
0 \\
0\end{array}$ & 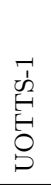 & 䆓 & 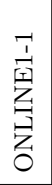 & 吉 & 窇 \\
\hline JAPIO-1 & $\gg$ & $\gg$ & $\gg$ & $\gg$ & $\gg$ & $\gg$ & $\gg$ & $\gg$ & $\gg$ & $\gg$ & $\gg$ & $\gg$ & $\gg$ & $\gg$ & $\gg$ & $\gg$ & $\gg$ & $\gg$ \\
\hline RBMT1-1 & & $>$ & $\gg$ & $\gg$ & $\gg$ & $\gg$ & $\gg$ & $\gg$ & $\gg$ & $\gg$ & $\gg$ & $\gg$ & $\gg$ & $\gg$ & $\gg$ & $\gg$ & $\gg$ & $\gg$ \\
\hline EIWA-1 & & & $\gg$ & $\gg$ & $\gg$ & $\gg$ & $\gg$ & $\gg$ & $\gg$ & $\gg$ & $\gg$ & $\gg$ & $\gg$ & $\gg$ & $\gg$ & $\gg$ & $\gg$ & $\gg$ \\
\hline RBMT3-1 & & & & - & $\gg$ & $\gg$ & $\gg$ & $\gg$ & $\gg$ & $\gg$ & $\gg$ & $\gg$ & $\gg$ & $\gg$ & $\gg$ & $\gg$ & $\gg$ & $\gg$ \\
\hline RBMT2-1 & & & & & $\gg$ & $\gg$ & $\gg$ & $\gg$ & $\gg$ & $\gg$ & $\gg$ & $\gg$ & $\gg$ & $\gg$ & $\gg$ & $\gg$ & $\gg$ & $\gg$ \\
\hline NTT-UT-1 & & & & & & - & - & - & - & $>$ & $\gg$ & $\gg$ & $\gg$ & $\gg$ & $\gg$ & $\gg$ & $\gg$ & $\gg$ \\
\hline TORI-1 & & & & & & & - & - & - & $\gg$ & $\gg$ & $\gg$ & $\gg$ & $\gg$ & $\gg$ & $\gg$ & $\gg$ & $\gg$ \\
\hline RWTH-1 & & & & & & & & - & - & $>$ & $\gg$ & $\gg$ & $\gg$ & $\gg$ & $\gg$ & $\gg$ & $\gg$ & $\gg$ \\
\hline BASELINE1-1 & & & & & & & & & - & - & $\gg$ & $\gg$ & $\gg$ & $\gg$ & $>$ & $\gg$ & $\gg$ & $\gg$ \\
\hline NAIST-1 & & & & & & & & & & - & $>$ & - & $>$ & $\gg$ & $>$ & $\gg$ & $\gg$ & $\gg$ \\
\hline FRDC-1 & & & & & & & & & & & - & - & $\gg$ & $>$ & - & $\gg$ & $\gg$ & $\gg$ \\
\hline BASELINE2-1 & & & & & & & & & & & & - & - & - & - & - & - & $\gg$ \\
\hline KYOTO-2 & & & & & & & & & & & & & - & - & - & $\gg$ & $\gg$ & $\gg$ \\
\hline KYOTO-1 & & & & & & & & & & & & & & - & - & - & - & $\gg$ \\
\hline UOTTS-1 & & & & & & & & & & & & & & & - & - & - & $\gg$ \\
\hline NEU-1 & & & & & & & & & & & & & & & & - & - & $\gg$ \\
\hline ONLINE1-1 & & & & & & & & & & & & & & & & & - & $\gg$ \\
\hline ICT-1 & & & & & & & & & & & & & & & & & & $\gg$ \\
\hline
\end{tabular}

of Japanese-English translation. On the other hand, the baseline commercial RBMT systems perform well for difficult word ordering of Japanese-English translations.

The results showing that RBMT systems were better than SMT systems were the same as the previous human evaluation results at NTCIR-7 (Fujii et al. 2008).

\subsubsection{Acceptability evaluation}

Table 10 shows the results of the acceptability evaluation. Table 11 shows the results of the statistical significance test of the acceptability evaluation using a sign test.

From the results, we can see that the source sentence meaning could be understood (C-rank and above) for $63 \%$ of the sentences in the best-ranked system using RBMT (JAPIO-1). For the best-ranked SMT system (NTT-UT-1), the source sentence meaning could be understood for $25 \%$ of the translated sentences (C-rank and above).

There was a large difference in the ability to retain the sentence-level meanings between the top-level commercial RBMT systems and the SMT systems for Japanese-to-English patent translation. 
Table 10 Results of JE acceptability.

\begin{tabular}{|c|c|c|c|c|c|c|c|c|c|c|}
\hline \multirow{2}{*}{ Run ID } & \multirow{2}{*}{ Type } & \multicolumn{3}{|c|}{ Resource } & \multirow{2}{*}{$\begin{array}{c}\text { Pairwise } \\
\text { score }\end{array}$} & \multicolumn{5}{|c|}{ Rate } \\
\hline & & $\mathrm{B}$ & $\mathrm{M}$ & $\mathrm{E}$ & & $\mathrm{AA}$ & A or higher & $\mathrm{B}$ or higher & $\mathrm{C}$ or higher & F or higher \\
\hline JAPIO-1 & RBMT & & & $\checkmark$ & 0.712 & 0.083 & 0.223 & 0.510 & 0.633 & 1.000 \\
\hline RBMT1-1 & RBMT & & & $\checkmark$ & 0.674 & 0.077 & 0.193 & 0.470 & 0.570 & 1.000 \\
\hline EIWA-1 & HYBRID & $\checkmark$ & & $\checkmark$ & 0.638 & 0.090 & 0.180 & 0.380 & 0.493 & 1.000 \\
\hline NTT-UT-1 & SMT & $\checkmark$ & & & 0.491 & 0.023 & 0.060 & 0.177 & 0.250 & 1.000 \\
\hline RWTH-1 & SMT & $\checkmark$ & & & 0.489 & 0.043 & 0.080 & 0.180 & 0.240 & 1.000 \\
\hline BASELINE1-1 & SMT & $\checkmark$ & & & 0.474 & 0.027 & 0.067 & 0.157 & 0.217 & 1.000 \\
\hline NAIST-1 & $\mathrm{SMT}$ & $\checkmark$ & & & 0.472 & 0.020 & 0.057 & 0.143 & 0.227 & 1.000 \\
\hline TORI-1 & SMT & $\checkmark$ & $\checkmark$ & $\checkmark$ & 0.460 & 0.043 & 0.063 & 0.123 & 0.183 & 1.000 \\
\hline FRDC-1 & $\mathrm{SMT}$ & $\checkmark$ & $\checkmark$ & & 0.448 & 0.017 & 0.040 & 0.113 & 0.187 & 1.000 \\
\hline BASELINE2-1 & SMT & $\checkmark$ & & & 0.447 & 0.037 & 0.060 & 0.123 & 0.167 & 1.000 \\
\hline KYOTO-1 & EBMT & $\checkmark$ & & & 0.436 & 0.007 & 0.027 & 0.103 & 0.177 & 1.000 \\
\hline UOTTS-1 & SMT & $\checkmark$ & & & 0.425 & 0.027 & 0.040 & 0.083 & 0.130 & 1.000 \\
\hline ONLINE1-1 & SMT & & & $\checkmark$ & 0.418 & 0.013 & 0.027 & 0.057 & 0.117 & 1.000 \\
\hline NEU-1 & SMT & $\checkmark$ & $\checkmark$ & & 0.416 & 0.017 & 0.023 & 0.060 & 0.120 & 1.000 \\
\hline
\end{tabular}

Table 11 Sign test of JE acceptability. "》": significantly different at $\alpha=0.01$, ">": significantly different at $\alpha=0.05$, "-": not significantly different at $\alpha=0.05$.

\begin{tabular}{|c|c|c|c|c|c|c|c|c|c|c|c|c|c|}
\hline & 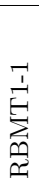 & $\sum_{\text {离 }}^{\stackrel{1}{\perp}}$ & 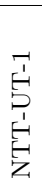 & 党 & 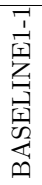 & 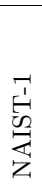 & $\frac{\overrightarrow{1}}{\overrightarrow{2}}$ & 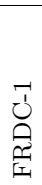 & $\begin{array}{l}\overline{1} \\
\text { 空 } \\
\text { 䛼 } \\
\text { 焉 }\end{array}$ & 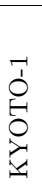 & 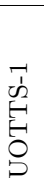 & $\begin{array}{l}\text { 辛 } \\
\text { 壱 } \\
\text { 学 }\end{array}$ & 怘 \\
\hline JAPIO-1 & - & $\gg$ & $\gg$ & $\gg$ & $\gg$ & $\gg$ & $\gg$ & $\gg$ & $\gg$ & $\gg$ & $\gg$ & $\gg$ & $\gg$ \\
\hline RBMT1-1 & & - & $\gg$ & $\gg$ & $\gg$ & $\gg$ & $\gg$ & $\gg$ & $\gg$ & $\gg$ & $\gg$ & $\gg$ & $\gg$ \\
\hline EIWA-1 & & & $\gg$ & $\gg$ & $\gg$ & $\gg$ & $\gg$ & $\gg$ & $\gg$ & $\gg$ & $\gg$ & $\gg$ & $\gg$ \\
\hline NTT-UT-1 & & & & - & - & - & - & $>$ & $\gg$ & $\gg$ & $\gg$ & $\gg$ & $\gg$ \\
\hline RWTH-1 & & & & & - & - & - & $\gg$ & $\gg$ & $\gg$ & $\gg$ & $\gg$ & $\gg$ \\
\hline BASELINE1-1 & & & & & & - & - & - & - & $>$ & $\gg$ & $\gg$ & $\gg$ \\
\hline NAIST-1 & & & & & & & - & - & - & - & $\gg$ & $\gg$ & $\gg$ \\
\hline TORI-1 & & & & & & & & - & - & - & $>$ & $\gg$ & $>$ \\
\hline FRDC-1 & & & & & & & & & - & - & - & - & $>$ \\
\hline BASELINE2-1 & & & & & & & & & & - & - & - & $>$ \\
\hline KYOTO-1 & & & & & & & & & & & - & - & $>$ \\
\hline UOTTS-1 & & & & & & & & & & & & - & - \\
\hline ONLINE1-1 & & & & & & & & & & & & & - \\
\hline
\end{tabular}

\subsection{English to Japanese}

\subsubsection{Adequacy evaluation}

Table 12 shows the results of the adequacy evaluation. Table 13 shows the results of the statistical significance test of the adequacy evaluation using a sign test.

NTT-UT-1 and NTT-UT-3 are the top systems for the SMT systems and RBMT6-1, JAPIO1, RBMT4-1, and RBMT5-1 are the top RBMT systems. From these results, the following are 
Table 12 Results of EJ adequacy.

\begin{tabular}{|c|c|c|c|c|c|c|c|c|c|c|}
\hline \multirow{2}{*}{ Run ID } & \multirow{2}{*}{ Type } & \multicolumn{3}{|c|}{ Resource } & \multirow{2}{*}{$\begin{array}{c}\text { Average } \\
\text { score }\end{array}$} & \multicolumn{5}{|c|}{ Rate } \\
\hline & & B & $\mathrm{M}$ & $\mathrm{E}$ & & 5 & 4 or higher & 3 or higher & 2 or higher & 1 or higher \\
\hline NTT-UT-1 & SMT & $\checkmark$ & $\checkmark$ & & 3.670 & 0.323 & 0.613 & 0.757 & 0.977 & 1.000 \\
\hline NTT-UT-3 & SMT & $\checkmark$ & & & 3.563 & 0.280 & 0.557 & 0.747 & 0.980 & 1.000 \\
\hline RBMT6-1 & RBMT & & & $\checkmark$ & 3.507 & 0.227 & 0.547 & 0.760 & 0.973 & 1.000 \\
\hline JAPIO-1 & RBMT & & & $\checkmark$ & 3.463 & 0.210 & 0.547 & 0.740 & 0.967 & 1.000 \\
\hline RBMT4-1 & RBMT & & & $\checkmark$ & 3.253 & 0.143 & 0.490 & 0.677 & 0.943 & 1.000 \\
\hline RBMT5-1 & RBMT & & & $\checkmark$ & 2.840 & 0.123 & 0.327 & 0.557 & 0.833 & 1.000 \\
\hline ONLINE1-1 & SMT & & & $\checkmark$ & 2.667 & 0.117 & 0.287 & 0.413 & 0.850 & 1.000 \\
\hline BASELINE1-1 & SMT & $\checkmark$ & & & 2.603 & 0.117 & 0.250 & 0.380 & 0.857 & 1.000 \\
\hline TORI-1 & HYBRID & $\checkmark$ & $\checkmark$ & $\checkmark$ & 2.600 & 0.050 & 0.260 & 0.470 & 0.820 & 1.000 \\
\hline BASELINE2-1 & SMT & $\checkmark$ & & & 2.477 & 0.107 & 0.203 & 0.327 & 0.840 & 1.000 \\
\hline KLE-1 & SMT & $\checkmark$ & & $\checkmark$ & 2.353 & 0.077 & 0.170 & 0.297 & 0.810 & 1.000 \\
\hline FRDC-1 & SMT & $\checkmark$ & $\checkmark$ & & 2.347 & 0.070 & 0.173 & 0.303 & 0.800 & 1.000 \\
\hline ICT-1 & SMT & $\checkmark$ & $\checkmark$ & & 2.320 & 0.053 & 0.150 & 0.287 & 0.830 & 1.000 \\
\hline UOTTS-1 & SMT & $\checkmark$ & & & 2.193 & 0.050 & 0.137 & 0.230 & 0.777 & 1.000 \\
\hline KYOTO-2 & SMT & $\checkmark$ & & & 2.180 & 0.077 & 0.137 & 0.233 & 0.733 & 1.000 \\
\hline KYOTO-1 & EBMT & $\checkmark$ & & & 2.047 & 0.050 & 0.133 & 0.260 & 0.603 & 1.000 \\
\hline BJTUX-1 & SMT & $\checkmark$ & & & 1.793 & 0.020 & 0.063 & 0.123 & 0.587 & 1.000 \\
\hline
\end{tabular}

Table 13 Sign test of EJ adequacy. "》": significantly different at $\alpha=0.01$, ">": significantly different at $\alpha=0.05$, "-": not significantly different at $\alpha=0.05$.

\begin{tabular}{|c|c|c|c|c|c|c|c|c|c|c|c|c|c|c|c|c|}
\hline & 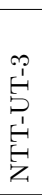 & 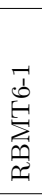 & 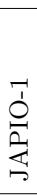 & $\sum_{\substack{n \\
\mid \vec{t}}}^{\vec{H}}$ & 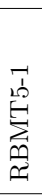 & 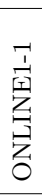 & 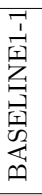 & $\frac{i}{\frac{1}{0}}$ & 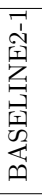 & $\begin{array}{l}\text { 岳 } \\
\text { 至 }\end{array}$ & 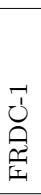 & 苞 & 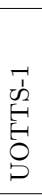 & 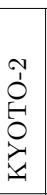 & 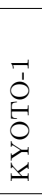 & 㟧 \\
\hline NTT-UT-1 & $\gg$ & - & $>$ & $\gg$ & $\gg$ & $\gg$ & $\gg$ & $\gg$ & $\gg$ & $\gg$ & $\gg$ & $\gg$ & $\gg$ & $\gg$ & $\gg$ & $\gg$ \\
\hline NTT-UT-3 & & - & - & $\gg$ & $\gg$ & $\gg$ & $\gg$ & $\gg$ & $\gg$ & $\gg$ & $\gg$ & $\gg$ & $\gg$ & $\gg$ & $\gg$ & $\gg$ \\
\hline RBMT6-1 & & & - & $\gg$ & $\gg$ & $\gg$ & $\gg$ & $\gg$ & $\gg$ & $\gg$ & $\gg$ & $\gg$ & $\gg$ & $\gg$ & $\gg$ & $\gg$ \\
\hline JAPIO-1 & & & & $\gg$ & $\gg$ & $\gg$ & $\gg$ & $\gg$ & $\gg$ & $\gg$ & $\gg$ & $\gg$ & $\gg$ & $\gg$ & $\gg$ & $\gg$ \\
\hline RBMT4-1 & & & & & $\gg$ & $\gg$ & $\gg$ & $\gg$ & $\gg$ & $\gg$ & $\gg$ & $\gg$ & $\gg$ & $\gg$ & $\gg$ & $\gg$ \\
\hline RBMT5-1 & & & & & & - & $>$ & $\gg$ & $\gg$ & $\gg$ & $\gg$ & $\gg$ & $\gg$ & $\gg$ & $\gg$ & $\gg$ \\
\hline ONLINE1-1 & & & & & & & - & - & $>$ & $\gg$ & $\gg$ & $\gg$ & $\gg$ & $\gg$ & $\gg$ & $\gg$ \\
\hline BASELINE1-1 & & & & & & & & - & - & $\gg$ & $\gg$ & $>$ & $\gg$ & $\gg$ & $\gg$ & $\gg$ \\
\hline TORI-1 & & & & & & & & & - & $\gg$ & $\gg$ & $\gg$ & $\gg$ & $\gg$ & $\gg$ & $\gg$ \\
\hline BASELINE2-1 & & & & & & & & & & - & - & - & $\gg$ & $\gg$ & $\gg$ & $\gg$ \\
\hline KLE-1 & & & & & & & & & & & - & - & - & $\gg$ & $\gg$ & $\gg$ \\
\hline FRDC-1 & & & & & & & & & & & & - & - & $\gg$ & $\gg$ & $\gg$ \\
\hline ICT-1 & & & & & & & & & & & & & $>$ & $\gg$ & $\gg$ & $\gg$ \\
\hline UOTTS-1 & & & & & & & & & & & & & & - & $\gg$ & $\gg$ \\
\hline KYOTO-2 & & & & & & & & & & & & & & & $>$ & $\gg$ \\
\hline KYOTO-1 & & & & & & & & & & & & & & & & $>$ \\
\hline
\end{tabular}


observed:

- The top SMT systems NTT-UT-1 and NTT-UT-3 achieved human evaluation scores (adequacy) that were equal to or better than the top-level commercial RBMT systems. This was not the case for any SMT system at NTCIR-7, and it is believed to be the first time that this is being achieved.

- The adequacy scores for the commercial RBMT systems were higher than those for SMT systems other than NTT-UT-1 and NTT-UT-3.

English-to-Japanese translation is difficult for SMT because the English and Japanese word order is significantly different. However, the top SMT systems achieved results equal to or better than the RBMT systems. There was one feature in the top SMT systems that improved translation quality. This feature, used in NTT-UT-1 and NTT-UT-3 (Sudoh et al. 2011) ${ }^{10}$, is that the systems utilize a method that pre-orders English input sentences using parse results and head finalization rules (Isozaki, Sudoh, Tsukada, and Duh 2010b) and then translates in almost monotone word orders. Since NTT-UT-1 uses a combination of three MT systems (two preordering systems and one forest-to-string system), the effectiveness of the pre-ordering method was not clear from just the NTT-UT-1 evaluation result. However, NTT-UT-3 consisted of only an MT system with the pre-ordering method using the head finalization rules. This allowed the effectiveness of the pre-ordering method using the head finalization rules to be seen from the results.

\subsubsection{Acceptability evaluation}

Table 14 shows the results of the acceptability evaluation. Table 15 shows the results of the statistical significance test of the acceptability evaluation using a sign test.

For the best SMT system (NTT-UT-1), the source sentence meaning could be understood ( $\mathrm{C}$ and above) for $60 \%$ of the sentences. Of the systems using RBMT, the source sentence meaning could be understood ( $\mathrm{C}$ or above) for $60 \%$ of the translated sentences in the best system (RBMT6-1).

The translation quality of the top SMT system (NTT-UT-1) was equal to or better than that of the top-level commercial RBMT systems for retaining the sentence-level meanings.

\footnotetext{
10 The NTT-UT system for Japanese-to-English translation also used a pre-ordering method. However, the system did not achieve an improvement as in English-to-Japanese translation because the pre-ordering method was different from the pre-ordering method for English-to-Japanese translation. The head finalization rules cannot be applied to Japanese-to-English translation since English is not a head final language.
} 
Table 14 Results of EJ acceptability.

\begin{tabular}{l|l|l|l|l|l|l|c|c|c|c}
\hline \multirow{2}{*}{ Run ID } & \multirow{2}{*}{ Type } & \multicolumn{2}{|c|}{ Resource } & Pairwise & \multicolumn{6}{c}{ Rate } \\
\cline { 5 - 9 } & & B & M & E & score & AA & A or higher & B or higher & C or higher & F or higher \\
\hline NTT-UT-1 & SMT & $\checkmark$ & $\checkmark$ & & 0.695 & 0.310 & 0.463 & 0.550 & 0.603 & 1.000 \\
RBMT6-1 & RBMT & & & $\checkmark$ & 0.656 & 0.147 & 0.410 & 0.500 & 0.600 & 1.000 \\
JAPIO-1 & RBMT & & & $\checkmark$ & 0.652 & 0.137 & 0.427 & 0.513 & 0.600 & 1.000 \\
ONLINE1-1 & SMT & & & $\checkmark$ & 0.479 & 0.060 & 0.193 & 0.250 & 0.293 & 1.000 \\
BASELINE1-1 & SMT & $\checkmark$ & & & 0.472 & 0.103 & 0.170 & 0.203 & 0.263 & 1.000 \\
BASELINE2-1 & SMT & $\checkmark$ & & & 0.456 & 0.103 & 0.153 & 0.190 & 0.227 & 1.000 \\
KLE-1 & SMT & $\checkmark$ & & $\checkmark$ & 0.434 & 0.063 & 0.127 & 0.157 & 0.193 & 1.000 \\
TORI-1 & SMT & $\checkmark$ & $\checkmark$ & $\checkmark$ & 0.432 & 0.047 & 0.117 & 0.157 & 0.203 & 1.000 \\
UOTTS-1 & SMT & $\checkmark$ & & & 0.411 & 0.057 & 0.090 & 0.113 & 0.157 & 1.000 \\
ICT-1 & SMT & $\checkmark$ & $\checkmark$ & & 0.411 & 0.033 & 0.093 & 0.130 & 0.177 & 1.000 \\
KYOTO-1 & EBMT & $\checkmark$ & & & 0.404 & 0.040 & 0.100 & 0.120 & 0.160 & 1.000 \\
\hline
\end{tabular}

Table 15 Sign test of EJ acceptability. "》": significantly different at $\alpha=0.01$, ">": significantly different at $\alpha=0.05$, "-": not significantly different at $\alpha=0.05$.

\begin{tabular}{|c|c|c|c|c|c|c|c|c|c|c|}
\hline & 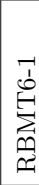 & 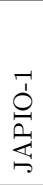 & $\begin{array}{l}\frac{1}{1} \\
\text { 公 } \\
\text { 夏 } \\
0\end{array}$ & $\begin{array}{l}\frac{1}{1} \\
\text { 空 } \\
\text { 空 } \\
\text { 离 }\end{array}$ & 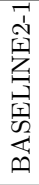 & 至 & 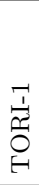 & 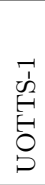 & $\overrightarrow{\underline{H}}$ & 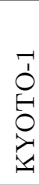 \\
\hline NTT-UT-1 & $>$ & $>$ & $\gg$ & $\gg$ & $\gg$ & $\gg$ & $\gg$ & $\gg$ & $\gg$ & $\gg$ \\
\hline RBMT6-1 & & - & $\gg$ & $\gg$ & $\gg$ & $\gg$ & $\gg$ & $\gg$ & $\gg$ & $\gg$ \\
\hline JAPIO-1 & & & $\gg$ & $\gg$ & $\gg$ & $\gg$ & $\gg$ & $\gg$ & $\gg$ & $\gg$ \\
\hline ONLINE1-1 & & & & - & - & $\gg$ & $\gg$ & $\gg$ & $\gg$ & $\gg$ \\
\hline BASELINE1-1 & & & & & - & - & - & $\gg$ & $\gg$ & $\gg$ \\
\hline BASELINE2-1 & & & & & & - & - & $>$ & $>$ & $\gg$ \\
\hline KLE-1 & & & & & & & - & - & - & - \\
\hline TORI-1 & & & & & & & & - & - & - \\
\hline UOTTS-1 & & & & & & & & & - & - \\
\hline ICT-1 & & & & & & & & & & - \\
\hline
\end{tabular}

\section{Validation of Human Evaluation Results}

To discuss reliability of the human evaluation, we present the correlation between the evaluation results for divided data. We validated the reliability of human evaluation as follows:

(1) The human evaluation data was divided into the first half data (Half-1) and the second half data (Half-2). Each contains half of all of the sentences evaluated by each evaluator.

(2) Scores for the systems based on the halved data were calculated.

(3) Correlation of system comparisons between the halved data was calculated.

Since the test data were built by random selection, it is assumed that the evaluation is not affected by differences in the halved data. Under this assumption, the following is true: If the evaluation 
is reliable, the top systems based on the first half data will also be the top systems based on the second half data, and the lower-ranking systems based on the first half data will also be the lower-ranking systems based on the second half data, i.e., there is good correlation between system comparison results of the two halved data. On the other hand, if the evaluation is not reliable, the top systems based on the first half data would be the lower-ranking systems based on the second half data, or the lower-ranking systems based on the first half data would be the top systems based on the second half data, i.e., there is poor correlation between system comparison results of the two halved data. Therefore, we validated the reliability based on the correlation between the evaluation results for the divided data. In this section, pairwise scores for systems were used for normalization purposes. A pairwise score for a system reflects the frequency with which it was judged to be better than or equal to other systems. A detailed explanation of the pairwise score is given in Section 2.2.2.

Figures 2-7 show the evaluation results for the first half of the data (Half-1), the second half of the data (Half-2), and all of the data (All). In the figures, the vertical axis is the pairwise score, and the horizontal axis is the Run ID. Although there are slight differences between the half data, there are no large differences that reverse the high-ranked and low-ranked systems.

Table 16 shows the Pearson correlation coefficients of the system evaluation scores between the half data. The Pearson correlation coefficients are close to 1.0 for all of the data pairs.

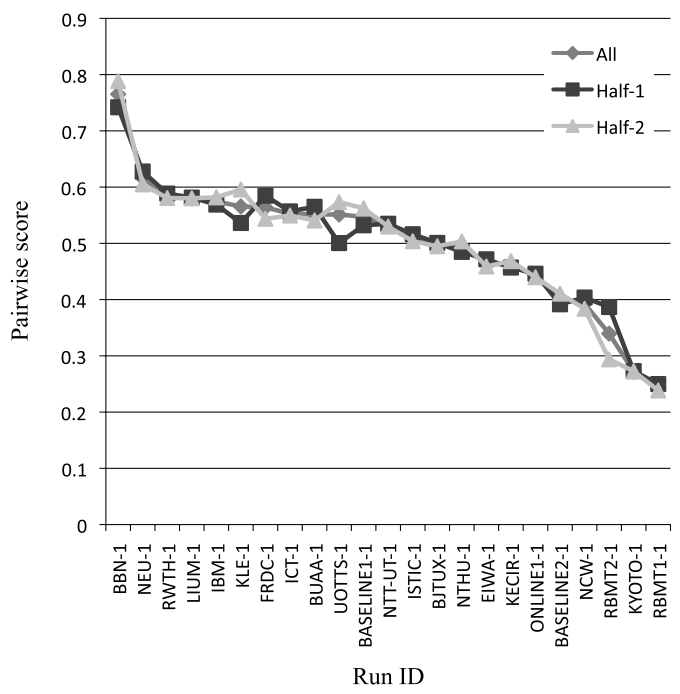

Fig. 2 Comparison between data for CE adequacy

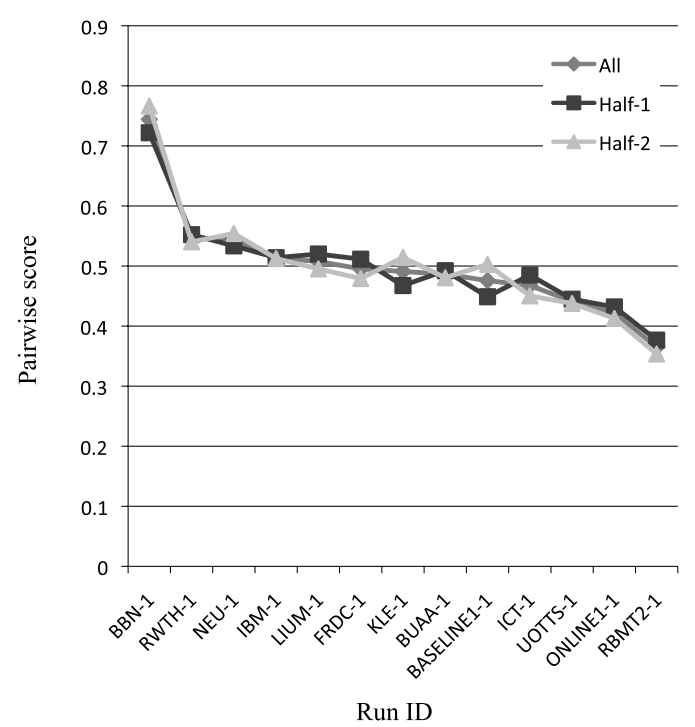

Fig. 3 Comparison between data for CE acceptability 


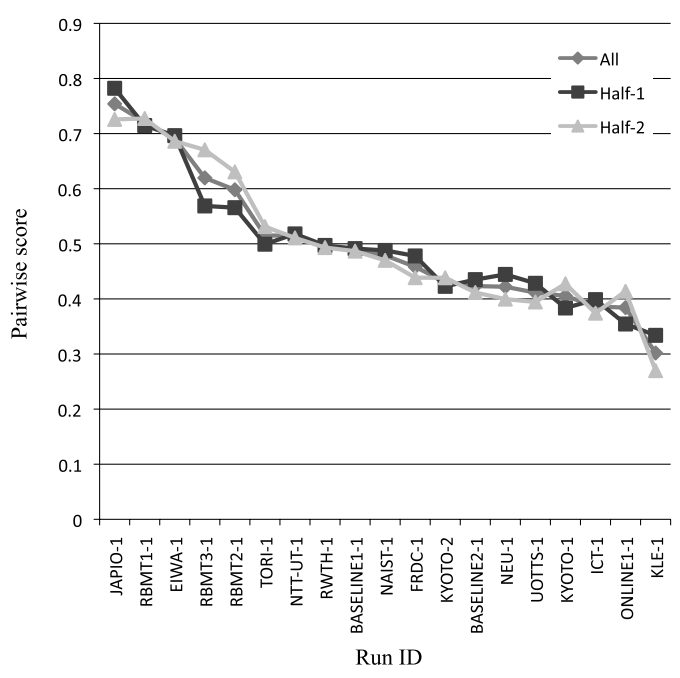

Fig. 4 Comparison between data for JE adequacy

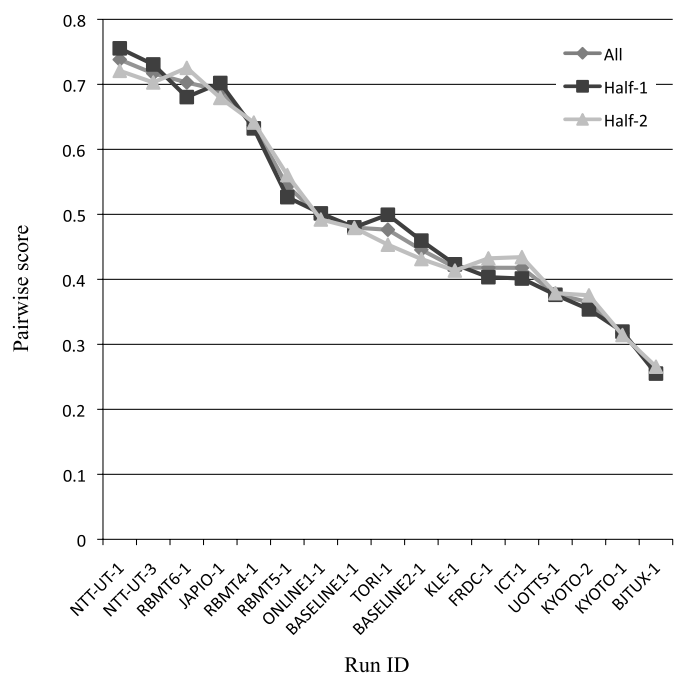

Fig. 6 Comparison between data for EJ adequacy

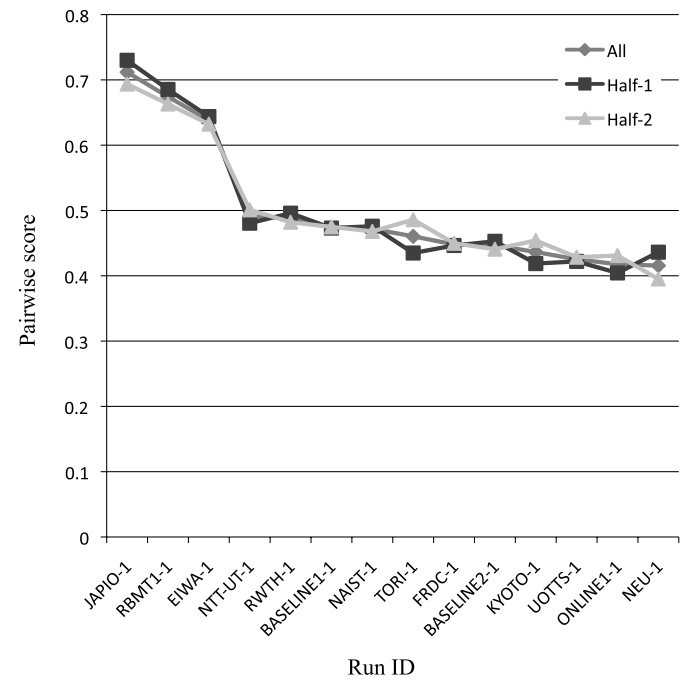

Fig. 5 Comparison between data for JE acceptability

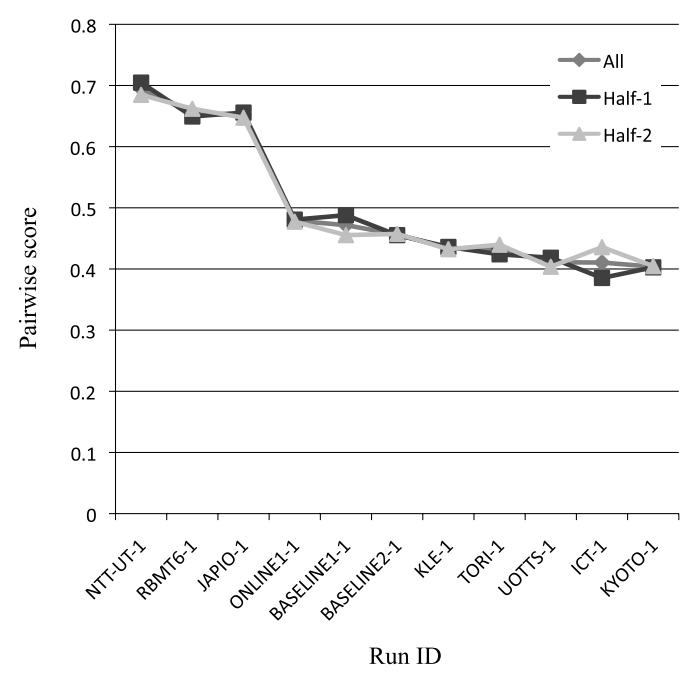

Fig. 7 Comparison between data for EJ acceptability

These indicate that the evaluations of 150 sentences are thought to be consistent for system comparison, and this consistency shows the reliability of the evaluation results. The evaluation results of 300 sentences are thought to be more reliable than the evaluation results of 150 sentences because the number of sentences is larger. 
Table 16 Pearson correlation coefficient between data.

\begin{tabular}{l|c|c}
\hline & Adequacy & Acceptability \\
\hline CE & 0.963 & 0.953 \\
JE & 0.940 & 0.972 \\
EJ & 0.985 & 0.982 \\
\hline
\end{tabular}

Table 17 Pearson correlation coefficient between evaluators by different data sets.

\begin{tabular}{c|c|c|c}
\hline & Evaluator & Adequacy & Acceptability \\
\hline CE & $1 \& 2$ & 0.929 & 0.918 \\
& $1 \& 3$ & 0.932 & 0.873 \\
& $2 \& 3$ & 0.966 & 0.898 \\
\hline JE & $1 \& 2$ & 0.944 & 0.967 \\
& $1 \& 3$ & 0.882 & 0.935 \\
& $2 \& 3$ & 0.945 & 0.966 \\
\hline EJ & $1 \& 2$ & 0.977 & 0.988 \\
& $1 \& 3$ & 0.963 & 0.979 \\
& $2 \& 3$ & 0.972 & 0.973 \\
\hline
\end{tabular}

In addition to the above main validation for reliability, we also checked the differences between evaluators. For each subtask and criterion, three evaluators evaluated the translations of 100 different source sentences. We checked the correlation between the evaluation results based on the 100 source sentences evaluated by the same evaluator. Table 17 shows the Pearson correlation coefficients for the system evaluation scores between evaluators. These values indicate that there is a high correlation between evaluators. Thus, even when the evaluators and the data are different, the evaluations are thought to be consistent for system comparison.

\section{Meta-Evaluation of the Automatic Evaluation Measure of BLEU}

We calculated the BLEU scores based on the 2,000 test sentences to investigate the reliability of the automatic evaluation measure of BLEU (Papineni et al. 2002), which is widely used to evaluate translation quality, in the patent domain for the language pairs of CE, JE, and EJ.

The Spearman rank-order correlation coefficients and the Pearson correlation coefficients between human evaluations (average adequacy scores) and the BLEU scores are shown in Table 18. From Table 18, it can be seen that the BLEU scores have a high correlation with the human evaluation for the $\mathrm{CE}$ evaluation, but do not have a high correlation with the human evaluation for the JE and EJ evaluations including RBMT systems. 
Table 18 Correlation coefficients between adequacy and the BLEU scores

\begin{tabular}{l|c|c}
\hline & Spearman & Pearson \\
\hline CE & 0.931 & 0.915 \\
JE & -0.042 & -0.241 \\
EJ & -0.029 & -0.032 \\
\hline
\end{tabular}

Table 19 Correlation coefficients between adequacy and the BLEU scores excluding RBMT systems

\begin{tabular}{c|c|c}
\hline & Spearman & Pearson \\
\hline JE & 0.618 & 0.525 \\
EJ & 0.511 & 0.753 \\
\hline
\end{tabular}

The Spearman rank-order correlation coefficients and the Pearson correlation coefficients between human evaluation and the BLEU scores excluding the RBMT systems for JE and EJ are shown in Table 19. The correlations excluding RBMT systems for JE and EJ are higher than those including the RBMT systems. Therefore, the reliability of the BLEU scores of the comparisons between systems without the RBMT systems is higher than that of the BLEU scores of the comparisons between systems including the RBMT systems for the automatic evaluation of the quality of the JE and EJ patent translations.

\section{Method for Obtaining the Database}

This section explains the method used to obtain resources. The available resources for research purposes consist of a human evaluation database, test data, reference data, and submission data (translated data). Resources are provided by the NTCIR project ${ }^{11}$. The method used to obtain the resources is given at the URLs shown in Table 20. Applicants are asked to sign a user agreement (memorandum on permission to use) to obtain the resources. The use of these resources is free of charge.

Table 20 URLs for obtaining the database.

\begin{tabular}{l|l}
\hline $\begin{array}{l}\text { Human evaluation } \\
\text { database and } \\
\text { test/reference data: }\end{array}$ & http://research.nii.ac.jp/ntcir/permission/ntcir-9/perm-en-PatentMT.html \\
\hline Submission data: & http://research.nii.ac.jp/ntcir/permission/rawdata-perm-en.html \\
\hline
\end{tabular}

11 http://research.nii.ac.jp/ntcir/ 


\section{Conclusion}

This paper presented information regarding the database of human evaluations from the NTCIR-9 Patent Machine Translation Task and the knowledge obtained from these evaluations. The evaluations showed the effectiveness of a number of machine translation systems in the patent translation field. Database of human evaluations is valuable for translation quality evaluation research. The resources will also be useful for system combination research. Resources including the human evaluation database, translation results, and test/reference data are available from the NTCIR project for research purposes.

\section{Acknowledgement}

We would like to thank all of the evaluators for constructing the database of human evaluations.

\section{Reference}

Callison-Burch, C., Koehn, P., Monz, C., Post, M., Soricut, R., and Specia, L. (2012). "Findings of the 2012 Workshop on Statistical Machine Translation." In Proceedings of the Seventh Workshop on Statistical Machine Translation, pp. 10-51, Montréal, Canada. Association for Computational Linguistics.

Chang, J., Huang, S.-T., Yen, H.-C., Jiang, M.-J., Huang, C.-C., Chang, J. S., and Yang, P.C. (2011). "[PatentMT] Summary Report of Team III_CYUT_NTHU." In Proceedings of NTCIR-9, pp. 684-688.

Chao, W. and Li, Z. (2011). "ZZX_MT: the BeiHang MT System for NTCIR-9 PatentMT Task." In Proceedings of NTCIR-9, pp. 629-633.

Ehara, T. (2011). "Machine translation system for patent documents combining rule-based translation and statistical post-editing applied to the PatentMT Task." In Proceedings of NTCIR9, pp. 623-628.

Feng, M., Schmidt, C., Wuebker, J., Peitz, S., Freitag, M., and Ney, H. (2011). "The RWTH Aachen System for NTCIR-9 PatentMT." In Proceedings of NTCIR-9, pp. 600-605.

Fujii, A., Utiyama, M., Yamamoto, M., and Utsuro, T. (2008). "Overview of the Patent Translation Task at the NTCIR-7 Workshop." In Proceedings of NTCIR-7, pp. 389-400.

Fujii, A., Utiyama, M., Yamamoto, M., Utsuro, T., Ehara, T., Echizen-ya, H., and Shimohata, S. 
(2010). "Overview of the Patent Translation Task at the NTCIR-8 Workshop." In Proceedings of NTCIR-8, pp. 371-376.

Goto, I., Lu, B., Chow, K. P., Sumita, E., and Tsou, B. K. (2011). "Overview of the Patent Translation Task at the NTCIR-9 Workshop." In Proceedings of NTCIR-9, pp. 559-578.

He, Y., Shi, C., and Wang, H. (2011). "ISTIC Statistical Machine Translation System for Patent Machine Translation in NTCIR-9." In Proceedings of NTCIR-9, pp. 634-637.

Hoang, H., Koehn, P., and Lopez, A. (2009). "A Unified Framework for Phrase-Based, Hierarchical, and Syntax-Based Statistical Machine Translation." In Proceedings of the International Workshop on Spoken Language Translation, pp. 152-159.

Isozaki, H., Hirao, T., Duh, K., Sudoh, K., and Tsukada, H. (2010a). "Automatic Evaluation of Translation Quality for Distant Language Pairs." In Proceedings of the 2010 Conference on Empirical Methods in Natural Language Processing, pp. 944-952.

Isozaki, H., Sudoh, K., Tsukada, H., and Duh, K. (2010b). "Head Finalization: A Simple Reordering Rule for SOV Languages." In Proceedings of the Joint Fifth Workshop on Statistical Machine Translation and MetricsMATR, pp. 244-251, Uppsala, Sweden. Association for Computational Linguistics.

Jiang, J., Xu, J., Lin, Y., and Zhang, Y. (2011). "System Description of BJTU-NLP SMT for NTCIR-9 PatentMT." In Proceedings of NTCIR-9, pp. 638-640.

Koehn, P., Hoang, H., Birch, A., Callison-Burch, C., Federico, M., Bertoldi, N., Cowan, B., Shen, W., Moran, C., Zens, R., Dyer, C., Bojar, O., Constantin, A., and Herbst, E. (2007). "Moses: Open Source Toolkit for Statistical Machine Translation." In Proceedings of the 45th Annual Meeting of the Association for Computational Linguistics Companion Volume Proceedings of the Demo and Poster Sessions, pp. 177-180.

Kondo, S., Komachi, M., Matsumoto, Y., Sudoh, K., Duh, K., and Tsukada, H. (2011). "Learning of Linear Ordering Problems and its Application to J-E Patent Translation in NTCIR-9 PatentMT." In Proceedings of NTCIR-9, pp. 641-645.

Lee, Y.-S., Xiang, B., Zhao, B., Franz, M., Roukos, S., and Al-Onaizan, Y. (2011). "IBM Chineseto-English PatentMT System for NTCIR-9." In Proceedings of NTCIR-9, pp. 606-613.

Lin, C.-Y. and Hovy, E. (2003). "Automatic Evaluation of Summaries Using N-gram Cooccurrence Statistics." In Proceedings of the 2003 Human Language Technology Conference of the North American Chapter of the Association for Computational Linguistics, pp. 71-78.

Lu, B., Tsou, B. K., Jiang, T., Kwong, O. Y., and Zhu, J. (2010). "Mining Large-scale Parallel Corpora from Multilingual Patents: An English-Chinese example and its application to SMT." In Proceedings of the 1st CIPS-SIGHAN Joint Conference on Chinese Language 
Processing (CLP-2010), Beijing, China.

Ma, J. and Matsoukas, S. (2011). "BBN's Systems for the Chinese-English Sub-task of NTCIR-9 Patent MT Evaluation." In Proceedings of NTCIR-9, pp. 579-584.

Murakami, J. and Tokuhisa, M. (2011). "Statistical Machine Translation with Rule based Machine Translation." In Proceedings of NTCIR-9, pp. 646-651.

Na, H., Li, J.-J., Kim, S.-J., and Lee, J.-H. (2011). "POSTECH's Statistical Machine Translation Systems for the NTCIR-9 PatentMT Task (English-to-Japanese)." In Proceedings of NTCIR9, pp. 652-656.

Nakazawa, T. and Kurohashi, S. (2011). "EBMT System of KYOTO Team in PatentMT Task at NTCIR-9." In Proceedings of NTCIR-9, pp. 657-660.

Ohio, T., Mitsuhashi, T., and Kakita, T. (2011). "Use of the Japio Technical Field Dictionaries for NTCIR-PatentMT." In Proceedings of NTCIR-9, pp. 614-617.

Papineni, K., Roukos, S., Ward, T., and Zhu, W.-J. (2002). "Bleu: a Method for Automatic Evaluation of Machine Translation." In Proceedings of 40th Annual Meeting of the Association for Computational Linguistics, pp. 311-318.

Pierce, J. R., Carroll, J. B., Hamp, E. P., Hays, D. G., Hockett, C. F., Oettinger, A. G., and Perlis, A. (1966). "Language and Machines: Computers in Translation and Linguistics." ALPAC report, National Academy of Sciences, National Research Council, Washington, DC.

Schwenk, H. and Abdul-Rauf, S. (2011). "LIUM's Statistical Machine Translation System for the NTCIR Chinese/English Patent Translation Task." In Proceedings of NTCIR-9, pp. 618-622.

Sudoh, K., Duh, K., Tsukada, H., Nagata, M., Wu, X., Matsuzaki, T., and Tsujii, J. (2011). "NTT-UT Statistical Machine Translation in NTCIR-9 PatentMT." In Proceedings of NTCIR-9, pp. 585-592.

Tseng, Y.-H., Liu, C.-L., Tsai, C.-C., Wang, J.-P., Chuang, Y.-H., and Jeng, J. (2011). "Statistical Approaches to Patent Translation for PatentMT - Experiments with Various Settings of Training Data." In Proceedings of NTCIR-9, pp. 661-665.

Utiyama, M. and Isahara, H. (2007). "A Japanese-English patent parallel corpus.” In Proceedings of Machine Translation Summit XI, Copenhagen, Denmark.

White, J. S., O'Connell, T. A., and O’Mara, F. E. (1994). "The ARPA MT Evaluation Methodologies: Evolution, Lessons, and Future Approaches." In Proceedings of AMTA.

Wu, X., Matsuzaki, T., and Tsujii, J. (2011). "SMT Systems in the University of Tokyo for NTCIR-9 PatentMT." In Proceedings of NTCIR-9, pp. 666-672.

Xiao, T., Li, Q., Lu, Q., Zhang, H., Ding, H., Yao, S., Xu, X., Fei, X., Zhu, J., Ren, F., and Wang, H. (2011). "The NiuTrans Machine Translation System for NTCIR-9 PatentMT." In 
Proceedings of NTCIR-9, pp. 593-599.

Xiong, H., Song, L., Meng, F., Lü, Y., and Liu, Q. (2011). "The ICT's Patent MT System Description for NTCIR-9." In Proceedings of NTCIR-9, pp. 673-678.

Zheng, Z., Ge, N., Meng, Y., and Yu, H. (2011). "HPB SMT of FRDC Assisted by Paraphrasing for the NTCIR-9 PatentMT." In Proceedings of NTCIR-9, pp. 679-683.

\section{Appendix}

\section{A Adequacy Criterion}

\section{A.1 Instructions for the Adequacy Criterion}

\section{A.1.1 Evaluation Criterion}

Adequacy is scored according to how well the meaning of a translation matches the meaning of the reference (source) translation for each sentence. Adequacy evaluations are done according to the 5-level scale shown in Table 21.

\section{A.1.2 Notes}

(1) Adequacy estimates the sentence meaning by evaluating fragments of a sentence.

(2) The main reason for using fragments is to reduce evaluation costs. When sentences are long, fragment-level evaluation is easier than sentence-level ones.

(3) Fragment size:

(a) Clause-level (first priority) or

(b) "subject and its predicate" level (second priority) or

(c) phrase-level (third priority).

(4) Supplementary definitions to reduce criterion ambiguity:

(a) A score of 5 indicates that the sentence-level meaning (subject, predicate and object) is correct.

Table 21 Adequacy criterion.

\begin{tabular}{l|l}
\hline 5 & All meaning \\
4 & Most meaning \\
3 & Much meaning \\
2 & Little meaning \\
1 & None \\
\hline
\end{tabular}


(b) Relative comparison:

- A sentence whose sentence-level meaning is not correct would be evaluated as 1-4 not only by the absolute criterion (most, much, little, and none) but also a relative comparison among the multiple translation outputs.

- The relative comparison must be consistent in all of the data.

\section{A.2 Example Values of Adequacy}

Examples of adequacy values and translations are shown in Table 22.

Table 22 Examples of adequacy values and translations.

\begin{tabular}{|c|c|c|}
\hline & Value & Sentence \\
\hline Source & & この基板ブラケットの詳細構成を図 8 に示す。 \\
\hline Reference & & The detailed constitution of the board bracket is shown in FIG. 8 . \\
\hline Translation & 5 & The detailed composition of this substrate bracket is shown in Fig. 8 . \\
\hline Source & & これによりセンスアンプ 18 からの出力を B L 2 に戻すことができる。 \\
\hline Reference & & By this, output of the sense amplifier 18 can be returned to BL2. \\
\hline Translation & 4 & $\begin{array}{l}\text { As a result, the output from the sense amplifier } 18 \text { can be returned to the BL } \\
\text { is } 2 \text {. }\end{array}$ \\
\hline Source & & 一般的には、端部ほどクラウニング落ち量が大きい。 \\
\hline Reference & & $\begin{array}{l}\text { Generally, the closer to the end point, the greater the amount of fall of the } \\
\text { crowning. }\end{array}$ \\
\hline Translation & 3 & Generally, the amount of clowning omissions is as large as an end. \\
\hline Source & & $\begin{array}{l}\text { 各々の二次電池 } 1 \text { を単独で順番に充電する組電池は、スイッチング回路 } 6 \text { のス } \\
\text { イッチング素子を利用して充電できる。 }\end{array}$ \\
\hline Reference & & $\begin{array}{l}\text { A battery assembly which sequentially charges each individual rechargeable bat- } \\
\text { tery } 1 \text { can utilize the switching devices of the switching circuitry } 6 \text { for charging. }\end{array}$ \\
\hline Translation & 2 & $\begin{array}{l}\text { Each of the secondary battery } 1 \text { to charge the battery can be charged by utilizing } \\
\text { a switching element of the switching circuit } 6 \text { is solely order }\end{array}$ \\
\hline Source & & $\begin{array}{l}\text { この場合も、隣り合う貫通孔 } 4 \text { との最小間隔が小さい貫通孔 } 4 \text { には、最小間隔が } \\
\text { 大きい貫通孔 } 4 \text { よりも少量の樹脂ペースト } 7 \text { を充填するようにする。 }\end{array}$ \\
\hline Reference & & $\begin{array}{l}\text { Also in this instance, the through holes } 4 \text { arranged at relatively smaller intervals } \\
\text { are filled with a smaller amount of resin paste } 7 \text { than the through holes } 4 \text { arranged } \\
\text { at relatively larger intervals. }\end{array}$ \\
\hline Translation & 1 & $\begin{array}{l}\text { Also in this case, the minimum interval is greater than the minimum interval } \\
\text { between adjacent through holes } 4 \text { is small, a small amount of resin paste } 7 \text { to } \\
\text { fill up the through hole } 4 \text { through hole } 4 \text {. }\end{array}$ \\
\hline
\end{tabular}




\section{B Acceptability Criterion}

\section{B.1 Instructions for the Acceptability Criterion \\ B.1.1 Evaluation Criterion}

Acceptability evaluations are done using the 5-level scale in Figure 1.

\section{B.1.2 Notes}

(1) Evaluations are performed from the perspective of whether the machine-translated English sentence conveys the important information and the content of the source sentence and not on the completeness of a literal translation.

(2) What is "important information"? "Important information" is the information that is necessary for a conversation between two people. This information is what needs to be conveyed by the machine translation results for the conversation partner to understand the content of the source sentence.

(3) What does "contents of the source sentence can be understood" mean? It refers to when two people can begin a conversation and the machine-translated results allow the conversation partner to understand the contents of the conversation.

(4) The first step and the second step of the chart can be merged; therefore, "F" means that either not all of the important information is included or the contents from the source sentence cannot be understood.

(5) The level of correctness for the "Grammatically correct" step indicates whether the translation is grammatical enough to convey the meaning of the source sentence. Strict adequateness (e.g., Editor's emendation level) for each expression is not required here. Therefore, if there are sentences that include expressions which cannot be considered to fully express the patent or technological terms, but the meaning itself is expressed, then it can be evaluated as A.

(6) On the "Native level" step, natural English sentences that do not need any correction are to be evaluated as AA. Therefore, all minimum required grammatical check points (including punctuation) for a natural English sentence are needed.

(7) If there is a sentence in unnatural English that lacks a subject (nominative), and if the sentence could be easily understood and is grammatically correct if it were transformed from the active sentence to the passive voice, it can be evaluated as "B," as the sentence is grammatically incorrect.

(8) The following type of differences is permissible: The character is the same but the character 
code is not the same. e.g., "1 23 " and " 123 " are considered to be the same.

(9) Special characters such as Greek letters in the source sentences are replaced as letters enclosed by periods or enclosed by ampersands and semicolons. These replacements are permissible. e.g., "5 $\mu$ m" $\rightarrow$ "5 .mu.m" or "5 \&mu;m"

(10) Some translations mistakenly include segments of characters from the source language. These segments are ignored if the translation works out appropriately without the segments.

\section{B.2 Example Values of Acceptability}

Examples of acceptability values and translations are shown in Table 23.

\section{Instructions for the Human Evaluation Procedure}

\section{C.1 Evaluation Method for Training and Main Evaluations}

- The criteria for evaluation are based on the guidelines.

- One input sentence (or one reference sentence) and all of the system outputs are shown simultaneously to compare systems.

- An evaluator evaluates all of the translations for the same input sentence.

- The MT output sentences for each input sentence are given to the evaluators in a random order.

Table 23 Examples of acceptability values and translations.

\begin{tabular}{|c|c|c|}
\hline & Value & Sentence \\
\hline Source & & この基板ブラケットの詳細構成を図 8 に示す。 \\
\hline Reference & & The detailed constitution of the board bracket is shown in FIG. 8 . \\
\hline Translation & AA & The detailed composition of this substrate bracket is shown in Fig. 8 . \\
\hline Source & & 変換されたディジタル音声データはA D P C Mアナライザ1 2 に入力される。 \\
\hline Reference & & The converted digital voice data is inputted into the ADPCM analyzer 12 . \\
\hline Translation & A & The changed digital voice data is inputted into the ADPCM analyzer 12. \\
\hline Source & & この基板ブラケットの詳細構成を図 8 に示す。 \\
\hline Reference & & The detailed constitution of the board bracket is shown in FIG. 8 . \\
\hline Translation & $\mathrm{B}$ & The substrate bracket detailed construction is shown in FIG . 8 . \\
\hline Source & & これらのガスは、所定の割合で混合して用いてもよい。 \\
\hline Reference & & These gases may be used in mixture in a prescribed proportion. \\
\hline Translation & $\mathrm{C}$ & These gases are mixed at a predetermined rate may be used \\
\hline Source & & 一般的には、端部ほどクラウニング落ち量が大きい。 \\
\hline Reference & & Generally, the closer to the end point, the greater the amount of fall of the crowning. \\
\hline Translation & $\mathrm{F}$ & Generally, the amount of clowning omissions is as large as an end. \\
\hline
\end{tabular}


- The evaluators can review the evaluations.

\section{C.2 Training}

Before the main evaluation, a trial evaluation is done. All of the evaluators evaluate translation results for the trial evaluations. The conditions for all evaluators are the same. After the trial evaluation, a consensus meeting is held to make corrections to the differences in the evaluations obtained from all of the evaluators and to decide on common evaluations for the translation results for the trial evaluation.

Isao Goto: received an M.E. in Electrical Engineering from Waseda University in 1997. He is a Research Expert at the National Institute of Information and Communications Technology (NICT). His research interests include machine translation.

Bin Lu: received an M.S. in Computer Science from Peking University in 2007. He is currently a $\mathrm{PhD}$ candidate at the Department of Chinese, Translation and Linguistics, City University of Hong Kong. He has published more than 20 academic papers in the area of Natural Language Processing and Computational Linguistics and holds two China patents. His research focuses on Statistical Machine Translation (especially in the patent domain) and Sentiment Analysis and Opinion Mining.

Ka Po Chow: received an MPhil in Electrical and Electronic Engineering in 2001. He is currently a project officer at the Research Centre on Linguistics and Language Information Sciences, Hong Kong Institute of Education, managing research projects and developing software in various areas including computational linguistics, multilingual sentence alignment, statistical machine translation and sentiment analysis. He has published a number of papers on parallel computing and natural language processing.

Eiichiro Sumita: received an M.S. in Computer Science from the University of Electro-Communications in 1982 and a Ph.D. in Engineering from Kyoto University in 1999. Dr. Sumita is the Director of the NICT Multilingual Trans- 
lation Laboratory. His research interests include machine translation and e-Learning.

Benjamin K. Tsou: received the MA degree from Harvard University, Cambridge, Massachusetts, and the Ph.D. degree from the University of California, Berkeley. He is a member of the Royal Academy of Overseas Sciences of Belgium and the chair professor of Linguistics and Asian Languages, as well as the director of the Language Information Sciences Research Center of the City University of Hong Kong. Since 1995, he has developed and cultivated the largest (350 million characters, 1.5 million word types by 2008) synchronous corpus of Chinese LIVAC (http://www.livac.org), which makes unique provisions for monitoring linguistic and related trends for application in NLP. He has authored several books and monographs, and more than 100 articles, mostly on computational linguistics and Chinese linguistics. He has served on the editorial boards of Natural Language Processing (Japan), the International Journal of Computational Linguistics and Chinese Language Processing (IJCLCLP) (Taipei), the International Journal of Computer Processing of Oriental Languages (Singapore), and the monograph series on natural language processing from John Benjamins (Amsterdam), among others. His current research interests include sentiment analysis, computational lexicography and lexicology, and resource developing and natural language processing. He is the founding president of the Asian Federation of Natural Language Processing (AFNLP) and was the chairman of SIGHAN, ACL. He also serves on the Executive Board of the Chinese Information Processing Society of China. He is a member of the IEEE.

Masao Utiyama: received a B.S. in Computer Science from the University of Tsukuba, Japan in 1992, an M.S. in Computer Science from the University of Tsukuba in 1994, a Ph.D. in Engineering from the University of Tsukuba in 1997. From 1997 to 1999, he was a Research Associate at Shinshu University, Japan. He has been a member of the National Institute of Information and Communications Technology (NICT), Japan since 1999. He is a Senior Researcher at NICT.

Keiji Yasuda: received an M.E. and a Ph.D. from Doshisha University in 2001 and 2004, respectively. He is currently a Senior Researcher at the National Institute of Communications Technology (NICT). His research interests include 
speech recognition, natural language processing, and e-Learning. He received a society paper award from the IEICE-ISS in 2006. He is a member of IEICE, IPSJ, and ASJ.

(Received October 9, 2012)

(Accepted November 25, 2012) 